\title{
The Chandrasekhar Theory of Stellar Collapse as the Limit of Quantum Mechanics
}

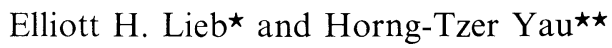 \\ Departments of Mathematics and Physics, Princeton University, P.O.B. 708, Princeton, \\ NJ 08544, USA
}

\section{Dedicated to Walter Thirring on his $60^{\text {th }}$ birthday}

\begin{abstract}
Starting with a "relativistic" Schrödinger Hamiltonian for neutral gravitating particles, we prove that as the particle number $N \rightarrow \infty$ and the gravitation constant $G \rightarrow 0$ we obtain the well known semiclassical theory for the ground state of stars. For fermions, the correct limit is to fix $G N^{2 / 3}$ and the Chandrasekhar formula is obtained. For bosons the correct limit is to fix $G N$ and a Hartree type equation is obtained. In the fermion case we also prove that the semiclassical equation has a unique solution - a fact which had not been established previously.
\end{abstract}

\section{Historical Remarks and Background}

There are two principal elementary models of stellar collapse: neutron stars and white dwarfs. In the former there is only one kind of particle which, since it is electrically neutral, interacts only gravitationally. The typical neutron kinetic energy is high, however, so it must be treated relativistically. Unfortunatly, the mass and density are also large enough that general relativistic effects are important. For white dwarfs, on the other hand, there are two kinds of nonneutral particles: electrons and nuclei. Because the density is not too large, it is a reasonable approximation to ignore general relativistic effects (although these effects might be important for stability considerations [29]); the nuclei (because of their large mass) can be treated nonrelativistically but the electrons must be treated relativistically. The Coulomb interaction is usually accounted for by the simple assumption that local neutrality requires the nuclear charge density to be equal to the electron charge density, in which case the problem reduces to calculating the electron density. (There are, in fact, electrostatic exchange and correlation effects [28, 29], but these are small by a factor $\alpha=1 / 137$.)

\footnotetext{
* Work partially supported by U.S. National Science Foundation grant PHY 85-15288-A 01
}

* Work supported by Alfred Sloan Foundation dissertation Fellowship 
Under the assumption of local neutrality (and no significant exchange and electron-nuclei correlation effects) and neglecting the nuclear kinetic energy, the white dwarf problem is mathematically the same as the neutron star problem - but without general relativistic effects. This problem was formulated by Chandrasekhar in 1931 [2] (and also in [7,11]) and leads to an equation for the density which we here call the Chandrasekhar equation $(1.16,1.18)$. The neutron star problem leads to the much more complicated Tolman-Oppenheimer-Volkoff equation which will not concern us. Both are reviewed in $[24,27]$. Both equations predict collapse at some critical mass which, in the white dwarf case, is called the Chandrasekhar mass. Clearly, near this mass the elementary theory is not totally adequate.

Quantum mechanics is essential for the stability in both cases. "The black-dwarf material is best likened to a single gigantic molecule in its lowest quantum state" [7]. In all treatments up to now, quantum mechanics enters only through the use of a local equation of state $P(\varrho),(P=$ pressure, $\varrho=$ density $)$ which is that of a degenerate Fermi gas (electrons or neutrons). See [30] for example.

Two years ago Lieb and Thirring [19] decided to investigate whether, starting from the Schrödinger equation for fermions one would, indeed, recover the semiclassical Chandrasekhar equation $(1.16,1.18)$ in the limit $N(=$ particle number $) \rightarrow \infty$ and $G(=$ gravitational constant $) \rightarrow 0$. More precisely, for fermions the relevant stability parameter should be $G N^{2 / 3}$, and not $G N$. Numerically, the critical $N$ is about $10^{57}$, so the limit $N \rightarrow \infty$ is a very reasonable one to consider. The Chandrasekhar value of the critical mass (with the correct $2 / 3$ exponent) was proved in [19], but only up to a factor of 4 . For bosons, on the other hand, which have not been considered for astrophysics, Ruffini and Bonazzola [30], Thirring [25], and Messer [21] realized that the relevant parameter should be $G N$, thus leading to collapse of objects only the size of a mountain. In [19] it was conjectured that, for bosons, (1.18) should be replaced by a Hartree type equation when $N \rightarrow \infty$. In a sense this would mean there is no semiclassical limit for bosons (although we shall continue to employ that word) because the Hartree energy involves density gradients, and not just an equation of state. In [19] the Hartree value of the collapse constant was proved to be correct up to a factor of 2 .

In this paper we shall prove that the Chandrasekhar (respectively Hartree) equations are exactly correct as $N \rightarrow \infty, G \rightarrow 0$, for all values of $G N^{2 / 3}$ (respectively $G N$ ), not just the critical value. In view of Walter Thirring's contributions to, and interest in quantum mechanical stability questions - in particular the stellar collapse problem - it is a great pleasure for us to dedicate this work to him on the occasion of his $60^{\text {th }}$ birthday.

At first it seemed to us that reducing the quantum problem to a semiclassical problem would end the story. But then we realized that a thorough mathematical study of (1.18), e.g. uniqueness of the solution, has not been done. This, it turn out, is in many ways more complicated than the quantum problem, and therefore a large part of this paper is devoted to an analysis of the semiclassical equations.

In Sect. I we state these problems precisely and summarize the main results. Section II contains proofs of the convergence of the quantum energies to the semiclassical energies. The analysis of the semiclassical equations (existence and uniqueness of solutions and qualitative properties) is in Sect. III and IV. The convergence of the quantum density (for fermions) to the semiclassical density is given in Sect. V. 


\section{Formulation of the Problem and Main Results}

Our starting point is the "relativistic" Schrödinger Hamiltonian for $N$ gravitating particles of mass $m$ (in units $\hbar=c=1$ )

$$
H_{\kappa N}=\sum_{i=1}^{N}\left\{\left(p_{i}^{2}+m^{2}\right)^{1 / 2}-m\right\}-\kappa \sum_{1 \leqq i<j \leqq N}\left|x_{i}-x_{j}\right|^{-1}
$$

Here $p^{2}=-\Delta$ and $x_{i} \in \mathbb{R}^{3} . H_{\kappa N}$ can describe a "neutron star" without general relativistic effects if we take $m=$ neutron mass and $\kappa=G m^{2}$. White dwarfs cannot be described by (1.1) (unless exchange and correlation effects are ignored). A more complicated Hamiltonian is needed in that case and we refer to [19, Sect. 4] for a discussion. Our methods can be extended to the case of several kinds of particles with different masses, but without electrostatic interaction. If electrostatic interactions are present, as in white dwarfs, genuinely new ideas are needed. However, if the positive nuclei are also fermions, then one can use the inequalities in [19, Sect. 4] to give a lower bound to the energy; unfortunately, this bound will not be the sharp one. It is believed that the semiclassical equation for white dwarfs is nearly the same as for (1.1) as $N \rightarrow \infty, G \rightarrow 0$ provided we take $\kappa=G(m+M / z)^{2}$ with $M=$ nuclear mass, $m=$ electron mass and $z=$ nuclear charge.

For fermions (e.g. neutrons or electrons) $H_{\kappa N}$ acts on antisymmetric functions of space and spin. For generality we assume $q$ spin states/particle; $q=2$ in nature, but $q=1$ would correspond to spin-polarized matter. We also consider $H_{\kappa N}$ without any symmetry restriction. Since the absolute ground state is always symmetric, this is the same as bosons (axion stars?). Technically, $H_{\kappa N}$ is considered as the Friedrich extension of the operator (1.1) with domain $\left\{\psi \in L^{2}\left(\mathbb{R}^{3 N}\right) \mid \psi\right.$ satisfies fermi statistics (or no statistics in boson case) and $\left(-\Delta_{i}\right)^{1 / 4} \psi \in L^{2}\left(\mathbb{R}^{2 N}\right)$ for $\left.i=1 \ldots N\right\}$.

The difficulty in going from $H_{\kappa N}$ to the semiclassical Chandrasekhar or Hartree theories as $N \rightarrow \infty$ and $G \rightarrow 0$ is this: For one particle, the operator $h=|p|-Z /|x|$ becomes unbounded below $[5,8-10,26]$ when $Z>2 / \pi$. Suppose that, by some fluctuation, $3(\pi \kappa)^{-1}$ particles get very close together. Then they form a trap into which the other particles can fall. Hence we might expect important correlation effects or even collapse for $H_{\kappa N}$ when $N=O\left(\kappa^{-1}\right)$, in which case the semiclassical point of view wherein the gravitational interaction is treated as a smooth perturbation would be wrong. Something like this does happen for bosons and that is why the Hartree equation is the appropriate limiting description. But the interesting (and difficult to prove) fact is that the Pauli principle prevents this from happening for fermions. There is a collapse in that case, but only when $N=O\left(\kappa^{-3 / 2}\right)$. The "local equation of state" point of view is valid for fermions.

The quantum energy is defined by

$$
E_{\kappa}^{Q}(N)=\inf \operatorname{spec} H_{\kappa N}
$$

in the appropriate space according to the statistics. Later on we shall define the quantum density.

The semiclassical energy functionals, $\mathscr{E}$ from $L^{1}\left(\mathbb{R}^{3}\right)$ to $\mathbb{R}$ are defined as follows. 
Fermions: For $t \in \mathbb{R}^{+}$, let $\eta=\left(6 \pi^{2} t / q\right)^{1 / 3}$ and

$$
\begin{aligned}
j(t) & \left.=q\left(2 \pi^{2}\right)^{-1} \int_{0}^{\alpha} p^{2}\left\{p^{2}+m^{2}\right)^{1 / 2}-m\right\} d p \\
& =q\left(16 \pi^{2}\right)^{-1}\left\{\eta\left(2 \eta^{2}+m^{2}\right)\left(\eta^{2}+m^{2}\right)^{1 / 2}-m^{4} \ln \left[\eta+\left(\eta^{2}+m^{2}\right)^{1 / 2}\right]\right\}-t m .
\end{aligned}
$$

Then

$$
\mathscr{E}_{\kappa}^{C}(\varrho) \equiv \int j(\varrho(x)) d x-\kappa D(\varrho, \varrho),
$$

where $D$ is the classical gravitational energy

$$
D(\varrho, \varrho)=\frac{1}{2} \iint \varrho(x) \varrho(y)|x-y|^{-1} d x d y .
$$

$j(\varrho)$ is the ground state kinetic energy density of $q$-state fermions at density $\varrho$.

Bosons:

$$
\mathscr{E}_{\kappa}^{H}(\varrho)=\left(\varrho^{1 / 2},\left\{\left(p^{2}+m^{2}\right)^{1 / 2}-m\right\} \varrho^{1 / 2}\right)-\kappa D(\varrho, \varrho) .
$$

The superscript $C$ is for Chandrasekhar while $H$ is for Hartree.

Corresponding to these functionals are the minimum energies:

$$
\begin{gathered}
E_{\kappa}^{C}(N)=\inf \left\{\mathscr{E}_{\kappa}^{C}(\varrho) \mid \varrho \geqq 0, \varrho \in L^{4 / 3}\left(\mathbb{R}^{3}\right) \text { and } \int \varrho=N\right\} \\
E_{\kappa}^{H}(N)=\inf \left\{\left.\mathscr{E}_{\kappa}^{H}(\varrho)|\varrho \geqq 0,| p\right|^{1 / 2} \varrho^{1 / 2} \in L^{2}\left(\mathbb{R}^{3}\right) \text { and } \int \varrho=N\right\}
\end{gathered}
$$

Later, we shall omit the subscript $\kappa$ when it is not necessary.

Recall (see [1, 19] and Lemma 3 for more details) that there is a critical constant $N_{f}(\kappa)$ which has the properties that $E_{\kappa}^{C}(N)=-\infty$ iff $N>N_{f}(\kappa) . N_{f}(\kappa)$ can be calculated explicitly. Define $\gamma \equiv \frac{3}{4}\left(6 \pi^{2} / q\right)^{1 / 3}$ and $\tau_{c} \equiv \gamma / \sigma_{f}$, where $\sigma_{f}=\sup \left\{D(\varrho, \varrho) / \int \varrho^{4 / 3} \mid \varrho \geqq 0, \varrho \in L^{4 / 3}\right.$ and $\left.\int \varrho=1\right\} \approx 1.092$ (see Appendix A). Then

$$
N_{f}(\kappa)=\tau_{c}^{3 / 2} \kappa^{-3 / 2} \approx 4.38 q^{-1 / 2} \kappa^{-3 / 2} .
$$

For bosons, there also exists a critical number $N_{b}(\kappa)$ which has the properties $E_{\kappa}^{H}(N)=-\infty$ iff $N>N_{b}(\kappa)$ (see [19] and Lemma 4). $N_{b}(\kappa)$ can be related to $\sigma_{b} \equiv \sup \left\{D(\varrho, \varrho) /\left.\left(\varrho^{1 / 2},|p| \varrho^{1 / 2}\right)|\varrho \geqq 0| p\right|^{,1 / 2} \varrho^{1 / 2} \in L^{2}\right.$ and $\left.\int \varrho=1\right\}$ by the formula

$$
N_{b}(\kappa)=\sigma_{b}^{-1} \kappa^{-1} \equiv \omega_{c} \kappa^{-1} \text {. }
$$

$\sigma_{b}$ is known to satisfy $\pi / 4>\sigma_{b}>1 / 2.7$ (Appendix A).

There are scalings

$$
E_{\kappa}^{C}(N)=t^{3 / 2} E_{t \kappa}^{C}\left(t^{-3 / 2} N\right), \quad E_{\kappa}^{H}(N)=t E_{t \kappa}^{H}\left(t^{-1} N\right)
$$

which are easy consequences of the transformation $\varrho(x) \rightarrow \varrho\left(t^{-1 / 2} x\right)$ (respectively $\left.\varrho(x) \rightarrow \varrho\left(t^{-1 / 3} x\right)\right)$. It is convenient to introduce some normalized quantities. For any $\tau>0$, let

$$
\begin{gathered}
\varepsilon_{\tau}^{c}(\varrho)=\int j(\varrho)-\tau D(\varrho, \varrho), \\
e^{c}(\tau)=\inf \left\{\varepsilon_{\tau}^{c}(\varrho) \mid \int \varrho=1, \quad \varrho \geqq 0 \text { and } \int \varrho^{4 / 3}<\infty\right\} .
\end{gathered}
$$

It is easy to see that [with $\left.\varrho(x) \equiv \varrho\left(N^{1 / 3} x\right)\right]$

$$
\mathscr{E}_{\kappa}^{C}(\varrho)=N \varepsilon_{\tau}^{c}(\varrho) \quad \text { and } \quad E_{\kappa}^{C}(N)=N e^{c}(\tau),
$$


where $\tau=N^{2 / 3} \kappa$. Similarly, we have (with $\omega=\kappa N$ )

$$
\mathscr{E}_{\kappa}^{H}(\varrho)=N \varepsilon_{\omega}^{H}(\varrho) \quad \text { and } \quad E_{\kappa}^{H}(N)=N e^{H}(\omega),
$$

where $\varepsilon_{\omega}^{H}$ and $e^{H}(\omega)$ are defined analogously to $(1.12,1.13)$.

Obviously, if we expect to have a nice limit as $N \rightarrow \infty$ and $G \rightarrow 0$ we should fix the quantities

$$
\tau \equiv \kappa N^{2 / 3} \text { (fermions) , } \omega \equiv \kappa N \text { (bosons) . }
$$

Numerically, $\kappa \approx 10^{-38}$ for neutrons or nuclei and $N$ is about $10^{57}$ for a neutron star or white dwarf, so this limit is quite justified physically.

Our main theorems can now be stated.

Theorem 1 (fermions). Fix $\tau=\kappa N^{2 / 3}$ and $q$ with $\tau<\tau_{c}$. Then

$$
\lim _{N \rightarrow \infty} E_{\kappa}^{Q}(N) / E_{\kappa}^{C}(N)=1 \text {. }
$$

If $\tau>\tau_{c}$ then $\lim _{N \rightarrow \infty} E_{\kappa}^{Q}(N)=-\infty$.

Theorem 2 (bosons). Fix $\omega=\kappa N$ with $\omega<\omega_{c}$. Then

$$
\lim _{N \rightarrow \infty} E_{\kappa}^{Q}(N) / E_{\kappa}^{H}(N)=1 \text {. }
$$

If $\omega>\omega_{c}$ then $\lim _{N \rightarrow \infty} E_{\kappa}^{Q}(N)=-\infty$.

Corollary 1. Let $N_{f}^{Q}(\kappa)$ [respectively $N_{b}^{Q}(\kappa)$ ] be the critical particle number for the stability of (1.1) in the fermion (respectively boson) case, i.e. $N^{Q}(\kappa)$ $=\sup \left\{N \mid E_{\kappa}^{Q}(N)>-\infty\right\}$. Then

$$
1=\lim _{\kappa \rightarrow 0} N_{f}^{Q}(\kappa) / N_{f}(\kappa)=\lim _{\kappa \rightarrow 0} N_{b}^{Q}(\kappa) / N_{b}(\kappa)
$$

if $q$ is fixed in the fermion case.

Remarks. (a) In fact, the errors between $E_{\kappa}^{Q}(N)$ and $E_{\kappa}^{C}(N)$ [respectively $E_{\kappa}^{H}(N)$ ] can be estimated (see Sect. II). The difference between the quantum and semiclassical critical particle numbers can be bounded for large $N$ as follows

$$
\begin{gathered}
\left(1+3 q^{2 / 9} N_{f}(\kappa)^{-2 / 9}\right) N_{f}(\kappa) \geqq N_{f}^{Q}(\kappa) \geqq\left(1-20 q^{1 / 9} N_{f}(\kappa)^{-1 / 9}\right) N_{f}(\kappa), \\
\left(1+2 N_{b}(\kappa)^{-1}\right) N_{b}(\kappa) \geqq N_{b}^{Q}(\kappa) \geqq\left(1-10 N_{b}(\kappa)^{-1 / 3}\right) N_{b}(\kappa) .
\end{gathered}
$$

(b) It was proved in [19] that $\lim N_{f}^{Q}(\kappa) / N_{f}(\kappa)$ is between 1 and $1 / 4$ (roughly). Likewise, $\lim N_{b}^{Q}(\kappa) / N_{b}(\kappa)$ is between 1 and $1 / 2$.

Theorems 1 and 2 show that we can study $H_{\kappa N}$ by means of its semiclassical approximations, (1.4) and (1.6), and therefore it behooves us to study the latter. Auchmuty and Beals [1] showed that there is a minimizing $\varrho$ for (1.4) for each $N<N_{f}$ and that this $\varrho$ has compact support. They did not prove uniqueness. Later, Lions [20, Theorem II.2] proved that any minimizing sequence of $\varrho$ 's for (1.4) has (after translation $\varrho(x) \rightarrow \varrho(x+y)$ ) a strongly convergent subsequence in $L^{4 / 3}\left(\mathbb{R}^{3}\right) \cap L^{1}\left(\mathbb{R}^{3}\right)$. 
The next two theorems summarize some properties of $E_{\kappa}^{C}(N)$ and $E_{\kappa}^{H}(N)$ which were not previously known, but which are physically important. For them, we need the notion of symmetric decreasing functions and rearrangements. For the convenience of the reader, we collect some basic definitions and facts about this subject in Appendix A. Since the functions we are interested in are all symmetric decreasing, we shall abuse notation by writing, say, $\varrho(r), \varrho(r) \equiv d \varrho / d r$, etc. with $r \equiv|x|$ for a function $\varrho: \mathbb{R}^{3} \rightarrow \mathbb{R}$.

Theorem 3 (fermions). (a) For each $N<N_{f}$, there exists a symmetric decreasing minimizer $\varrho_{N}(x)$ for $E_{\kappa}^{C}(N)$. It satisfies the Euler-Lagrange equation for some Lagrange multiplier $\mu$ :

$$
j^{\prime}(\varrho(x))=\left[\eta^{2}(x)+m^{2}\right]^{1 / 2}-m=\left\{\kappa|x|^{-1} * \varrho-\mu\right\}_{+},
$$

where $\{f(x)\}_{+} \equiv \max (f(x), 0)$ and $\eta(x)=\left(6 \pi^{2} \varrho(x) / q\right)^{1 / 3}$.

(b) Any minimizing $\varrho$ for $E_{\kappa}^{C}(N)$ is symmetric decreasing after translation and satisfies (1.16) for some $\mu$.

(c) There is no minimizing @ for $E_{\kappa}^{C}\left(N_{f}\right)$ even though $E_{\kappa}^{C}\left(N_{f}\right)$ is finite.

(d) $E_{\kappa}^{C}(N)$ is a strictly concave, monotone decreasing function which is continuous at the end point, $N_{f}$, and $E_{\kappa}^{C}\left(N_{f}\right)=-m N_{f}$.

(e) Let $\mu_{N}$ be the Lagrange multiplier associated to some minimizer $\varrho_{N}$ for $N<N_{f}$. Then the right and left derivatives of $E_{\kappa}^{C}(N)$ satisfy $\left(d E_{\kappa}^{C} / d N\right)_{+} \leqq \mu_{N} \leqq\left(d E_{\kappa}^{C} / d N\right)_{-}$.

(f) $\mu_{N} \rightarrow \infty$ as $N \rightarrow N_{f}$.

Remarks. (a) The Euler-Lagrange equation (1.16) is in fact equivalent to the Newtonian limit of the Tolman-Oppenheimer-Volkoff equation ((11.1.13) and (11.3.4) of [27], see also [24]). By differentiating (1.16) with respect to $r$ we have

$$
\begin{aligned}
\dot{\varrho} j^{\prime \prime}(\varrho) & =\frac{2 \pi^{2}}{q}\left[\eta^{2}(r)+m^{2}\right]^{-1 / 2} \eta(r)^{-1} \varrho(r)=-\kappa M(r) / r^{2}, \\
M(r) & =4 \pi \int_{0}^{r} s^{2} \varrho(s) d s .
\end{aligned}
$$

Let $P(r)=\frac{q}{6 \pi^{2}} \int_{0}^{\alpha(r)} k^{4}\left(k^{2}+m^{2}\right)^{-1 / 2} d k$ be the pressure ((11.3.43) of [27]). Then (1.17) can be rewritten as an equation of gravitational-hydrostatic equilibrium:

$$
-r^{2} \dot{P}(r)=\kappa M(r) \varrho(r) .
$$

Equation (1.18) is the Newtonian limit of the TOV equation. For historical reasons, we call (1.16), and its equivalent (1.18), the Chandrasekhar equation.

(b) The Euler-Lagrange equation for (1.4) is really $j^{\prime}(\varrho)-\kappa|x|^{-1} * \varrho-\mu=0$ when $\varrho(x)>0$ and $\geqq 0$ when $\varrho(x)=0$. But, since $j^{\prime}(0)=0$, this is equivalent to (1.16).

(c) (1.16) is equivalent to a second order partial differential equation. See (4.7) and Lemma 8.

(d) Theorem 5(b) improves Theorem 3(e).

Theorem 4 (bosons). (a) For each $N<N_{b}$, there exists a symmetric decreasing minimizer $\varrho_{N}(x)$ for $E_{\kappa}^{H}(N)$. It satisfies the Euler-Lagrange equation with Lagrange 


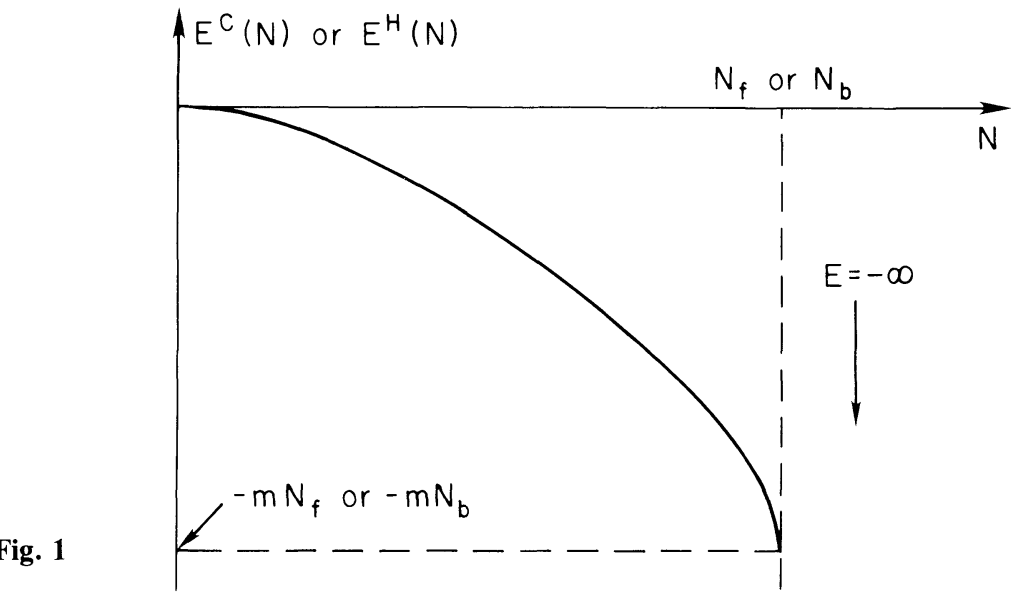

multiplier $v$ in the distributional sense (with $\left.p^{2}=-\Delta\right)$ :

$$
\left[\left(p^{2}+m^{2}\right)^{1 / 2}-m\right] \varrho^{1 / 2}=\left(\kappa|x|^{-1} * \varrho-v\right) \varrho^{1 / 2} .
$$

(b), (c), (d), (e), (f) Same as in Theorem 3 mutatis mutandis.

Figure 1, which is schematic, summarizes parts of Theorems 3 and 4.

The fact that $\mathscr{E}_{\kappa}^{C}(\varrho)$ has a local kinetic energy enables us to study $E_{\kappa}^{C}(N)$ in more detail. In the next theorem, we show that $E_{\kappa}^{C}(N)$ has a unique minimizer up to translation for $N<N_{f}$. We also show that the central density is strictly increasing to infinity while the radius is strictly decreasing to zero as $N \rightarrow N_{f}$.

The next two theorems are stated only for fermions. While we do not expect that their analogues fail for bosons, to prove them would require a great deal more work. It will be time enough to undertake this work when boson stars are seen in the sky.

Theorem 5 (fermions). (a) For each $N<N_{f}$ the minimizer $\varrho_{N}$ is unique up to translations $\varrho(x) \rightarrow \varrho(x+y)$ for $y \in \mathbb{R}^{3}$.

(b) $E_{\kappa}^{C}(N)$ is differentiable in $N$ and thus $d E_{\kappa}^{C}(N) / d N=-\mu_{N}$.

(c) Each $\varrho_{N}$ has compact support. Let $R_{N}$ denote the radius of its support. $\varrho_{N}(r)$ is real analytic for $r<R_{N}$. $R_{N}$ is a strictly decreasing function of $N . R_{N} \rightarrow 0$ as $N \rightarrow N_{f}$ and $R_{N} \rightarrow \infty$ as $N \rightarrow 0$.

(d) Let $\alpha_{N} \equiv \varrho_{N}(0)$ be the central density of $\varrho_{N}$. Then $\alpha_{N}$ is a strictly increasing function of $N$ tending to $\infty$ as $N \rightarrow N_{f}$.

(e) Every radial solution of (1.16) is a minimizer for $N=\int \varrho$.

(f) Any two $\varrho_{N}$ 's intersect at exactly one value of $r$.

(g) If $N_{1}<N_{2}$ then $M_{1}(r)<M_{2}(r)$ for all $r>0$ [M(r) is defined in (1.17)].

Some of these results are displayed schematically in Fig. 2.

Remarks. (a) We can relate Theorem 5 to the stability theorem given in e.g. [24, 27]. Since the Euler-Lagrange equation is equivalent to the Chandrasekhar equation, Theorem 5 asserts that any solution of the Chandrasekhar equation with given central density is an absolute (global) minimizer for some $E^{C}(N)$ or, in other words, is stable. However, our result is stronger than the standard result in $[24,27]$ where only local stability is discussed. 
Fig. 2

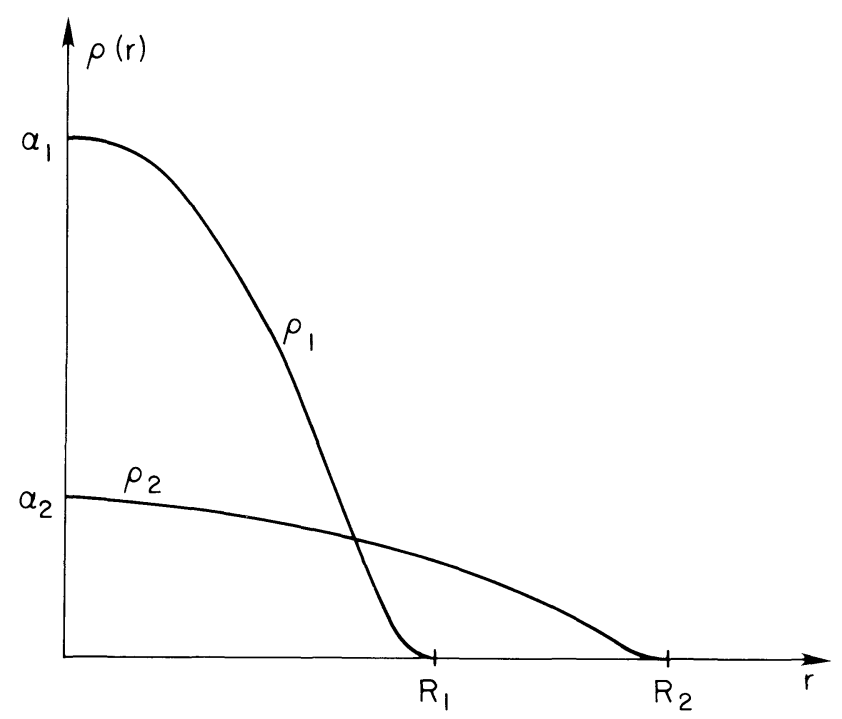

(b) The normalized semiclassical functional $\varepsilon_{\tau}^{c}$ has properties similar to $\mathscr{E}_{\kappa}^{C}$. In particular, $e^{c}(\tau)$ has a unique solution $\varrho_{\tau}$ (say). It is easy to check from the scaling that $\varrho_{N}(x)=\varrho_{\tau}\left(N^{-1 / 3} x\right)$.

(c) As will become clear, the uniqueness (up to translations) of the solution amounts to the fact that the radius and central density of the star is a continuous function of the particle number. There are no "phase transitions". While it is clear that the radius is a continuous function of the central density $\alpha$, it is not obvious that there are some $\alpha$ 's which do not correspond to some minimizer. If that were to happen (and we shall show that it does not) then $\alpha$ (and hence the radius) would not be continuous in $N$.

Equation (1.16) is equivalent to a PDE as shown in (4.7). [Actually, the fact that every solution to (4.7) is a solution to (1.16) is not obvious; in Lemma 8 we prove this for radial solutions.] Theorem 5 translates into statements about the ODE arising from (4.7) in the radial case. This kind of ODE was investigated in an important paper of $\mathrm{Ni}$ [23]. He proves that $R(\alpha)$ is a decreasing function of the central density, $\alpha$, [cf. Theorem 5(c) and (d)] but his uniqueness problem is different from ours since he fixes $R$ while we fix $N$. It is possible to use Ni's result to prove Theorem 5 [except for $(\mathrm{g})$ ]. We originally followed that route and found the proof to be quite complicated. The proof we actually present here is, in our opinion, much more direct. It uses the variational principle in an essential and, we believe, novel way. Admittedly the class of ODE's that can be treated by our method is more restricted than Ni's.

We turn now to the connection between the ground state of $H_{\kappa N}$ and the unique semiclassical density $\varrho_{N}$ that minimizes $\mathscr{E}_{\kappa}^{C}$. Recall that $\varrho_{N}(x)=\varrho_{\tau}\left(N^{-1 / 3} x\right)$, where $\varrho_{\tau}$ minimizes $\varepsilon_{\tau}^{c}$ and is independent of $N$, and $\tau=\kappa N^{2 / 3}$. Given a normalized $N$ particle function, $\psi$, of space-spin we define the one-particle density as

$$
\varrho_{\psi}(x)=N \sum_{\sigma} \int\left|\psi\left(x, x_{2}, \ldots, x_{N} ; \sigma_{1}, \ldots, \sigma_{N}\right)\right|^{2} d x_{2} \ldots d x_{N} .
$$


(A similar definition holds for bosons without $\sum_{\sigma}$.) In some sense, if $\psi_{N}$ is a "ground state" of $H_{\kappa N}$, and if $\varrho_{N}^{Q}$ is the density defined by (1.20) with $\psi_{N}$, we expect that $\varrho_{N}^{Q}\left(N^{1 / 3} x\right)$ should converge to $\varrho_{\tau}(x)$ as $N \rightarrow \infty$. There are several conceptual difficulties with this expectation as we now explain.

For one thing $H_{\kappa N}$ is translation invariant so it has no $L^{2}$ eigenfunction. For this reason, and also because it is physically sensible to consider only functions $\psi$ which are "near" the ground state when the particle number is huge, we first have to introduce the concept of an approximate ground state as in [18].

Definition 1. Fix $\tau$. A sequence of normalized wave functions $\psi_{N}$ is said to be an approximate ground state if, as $N \rightarrow \infty$,

$$
N^{-1}\left|\left(\psi_{N}, H_{\kappa N} \psi_{N}\right)-E_{N}^{Q}\right| \rightarrow 0 \text {. }
$$

Even with this definition there is another problem. In quantum mechanics the fact that $\psi_{N}$ has a low energy does not imply that the system is localized. To see this, let $g(x)$ be any nonnegative function with $\int g=1$, and define the density matrix $\Gamma$ by $\Gamma\left(X, \sigma \mid X^{\prime} \sigma^{\prime}\right) \equiv \int g(y) \psi_{N}\left(x_{i}+y, \sigma\right) \psi_{N}\left(x_{i}^{\prime}+y, \sigma^{\prime}\right) d y$. One finds easily that the energy of $\Gamma, \operatorname{Tr} \Gamma H_{\kappa N}$, equals $\left(\psi_{N}, H_{\kappa N} \psi_{N}\right)$, but $\Gamma$ has a one-particle density given by $\varrho_{\Gamma}=\varrho_{N}^{Q} * g$. Thus the single-particle density can be smeared out as much as we please without affecting the energy.

The usual way out of this difficulty is to fix a certain relatively small number of particles and then to discuss the density of the rest. But it is clear that the number that have to be fixed is huge. Thus, this approach is difficult to implement.

What we do instead is to use a localizing potential. We take advantage of the fact that $\varrho_{\tau}(x)$ has support in a ball of some radius $d_{\tau}$. Let $\chi_{\tau}$ be the characteristic function of this ball and let $\lambda>0$. If we add $-\lambda \int \chi_{\tau} \varrho$ to $\varepsilon_{\tau}^{c}$, then the minimum energy would be exactly $e^{c}(\tau)-\lambda$ and the minimizer would be uniquely $\varrho_{\tau}$ [formerly all $\varrho_{\tau}(x+y), y \in \mathbb{R}$, were minimizers]. Let us do the same to (1.1)

$$
H_{\kappa N \lambda} \equiv H_{\kappa N}-\lambda \sum_{i=1}^{N} \chi_{\tau}\left(N^{-1 / 3} x_{i}\right) \text {. }
$$

Theorem 6. Fix $\lambda>0$ and $\tau=\kappa N^{2 / 3}$. Let $\psi_{N \lambda}$ be a sequence of approximate ground states for $H_{\kappa N \lambda}$ as in (1.21) and let $\varrho_{N \lambda}^{Q}(x)$ be the densities as in (1.20). Then, for all $\lambda>0$ and $N \rightarrow \infty$,

weakly in $L^{4 / 3} \cap L^{1}\left(\mathbb{R}^{3}\right)$.

$$
\varrho_{N \lambda}^{Q}\left(N^{1 / 3} x\right) \rightarrow \varrho_{\tau}(x)
$$

Remark. The specific choice of $\chi_{\tau}$ as a localizing potential is arbitrary. Any other potential for which it is possible to prove the uniqueness of the minimum for $\varepsilon_{\tau \lambda}^{c}$ would suffice.

\section{Convergence of the Quantum Energy to the Semiclassical Energy}

The easy part is the upper bound to $E^{Q}(N)$ and we shall dispose of that first. Our proof is basically the same as that in [19, pp. 503-508] which uses the variational principle with coherent states for fermions and a product state for bosons. Only the main points need be given here. 


\section{A.1. Upper Bound to the Quantum Energy (Fermions)}

To imitate the proof of [19, Theorem 2$]$ it is only necessary to verify the analogue of [19, Eqs. (45), (46)] for our kinetic energy, namely for all $k, p \in \mathbb{R}^{3}$

$$
\left.\left[k^{2}+m^{2}\right]^{1 / 2}-m \leqq|k+p|+\mid p^{2}+m^{2}\right]^{1 / 2}-m \text {. }
$$

[This follows from the triangle inequality by thinking of $(k, m),(p,-m)$ as two vectors in $\mathbb{R}^{4}$ with the Euclidean norm.] Then we deduce [19, Eq. (51)] that for every nonnegative $\bar{\varrho}(x)$ with $\int \bar{\varrho}=N$ and every $\xi>0$,

$$
E^{Q}(N) \leqq \mathscr{E}_{\kappa}^{C}(\bar{\varrho})+(1.68) \kappa \int \bar{\varrho}^{4 / 3}+2 N \xi^{1 / 2}+\xi^{-1} \kappa \int \bar{\varrho}^{2} .
$$

Choosing $\bar{\varrho}$ to be a minimizer, $\varrho$, for $\mathscr{E}_{\kappa}^{C}$ and optimizing $\xi$ we obtain

$$
E^{Q}(N) \leqq E^{C}(N)+(1.68) \tau N^{1 / 3} \int f^{4 / 3}+3 N^{7 / 9} \tau^{1 / 3}\left(\int f^{2}\right)^{1 / 3} .
$$

[Recall $\tau=\kappa N^{2 / 3}$ and $\varrho(x)=f\left(N^{-1 / 3} x\right)$.] Since $E^{C}(N)=N e^{c}(\tau)$ and $f \in L^{2} \cap L^{\infty}$ for a minimizer (cf. Sect. IV) when $\tau<\tau_{c}$, we see that (2.3) can be bounded for all $\tau<\tau_{c}$ as

$$
E^{Q}(N) \leqq E^{C}(N)\left(1-C_{1}(\tau, q) N^{-2 / 9}\right)
$$

for some function $C_{1}(\tau, q)$. Theorem 2 of [19] states that $E^{Q}(N)=-\infty$ when $\tau>\tau_{c}\left(1+C_{2} N^{-2 / 9}\right)$.

\section{A.2. Upper Bound to the Quantum Energy (Bosons)}

Again, we follow [19, p. 505]. Given $\bar{\varrho}(x) \geqq 0$ with $\int \bar{\varrho}=N$, we define the normalized variational function

$$
\psi\left(x_{1}, \ldots, x_{N}\right) \equiv N^{-N / 2} \prod_{j=1}^{N} \bar{\varrho}\left(x_{j}\right)^{1 / 2} .
$$

Then, adding and subtracting the self interaction, we get

$$
\left(\psi, H_{N} \psi\right)=\mathscr{E}^{H}(\bar{\varrho})+\frac{1}{2} \kappa N^{-1} D(\bar{\varrho}, \bar{\varrho}) .
$$

Choosing $\varrho$ to be a minimizer, $\varrho$, for $\mathscr{E}^{H}$ and recalling that $\omega=\kappa N, \varrho(x)=f\left(N^{-1} x\right)$, we obtain the analogue of (2.4) for all $\omega<\omega_{c}$ :

$$
E^{Q}(N) \leqq E^{H}(N)\left(1-C_{3}(\omega) N^{-1}\right) .
$$

When $\omega>[N /(N-1)] \omega_{c}, E^{Q}(N)=-\infty$.

Now we present the lower bound to $E^{Q}(N)$ which, apart from the analysis of the semiclassical equation, is the main mathematical point of this paper.

\section{B.1. Lower Bound to the Quantum Energy (Fermions)}

As in [19, Eq. (4)] we write $H_{N}$ as a sum of operators, but here the operators will be more complicated than in [19]. Let $P$ be a partition of $\{1, \ldots, N\}$ into two disjoint sets $\pi_{1}$ and $\pi_{2}$ of sizes $L$ and $M$ respectively, with $L+M=N$. There are $\left(\begin{array}{l}N \\ L\end{array}\right)$ such partitions. $X=\left\{x_{1}, \ldots, x_{N}\right\}$ denotes the $N$ variables in $\mathbb{R}^{3}$. 


$$
H_{N}=\left\{\frac{N}{L}\left(\begin{array}{l}
N \\
L
\end{array}\right)^{-1} \sum_{P} h_{P}\right\}+H^{(2)} .
$$

The $h_{P}$ and $H^{(2)}$ are given in terms of three positive parameters $e, \kappa^{\prime}, \varepsilon$ with $\varepsilon<1$ as

$$
\begin{aligned}
h_{P}= & (1-\varepsilon) \sum_{i \in \pi_{1}}\left\{\left(p_{i}^{2}+m^{2}\right)^{1 / 2}-m\right\}-\kappa^{\prime} e\left[\sum_{i \in \pi_{1}} \sum_{j \in \pi_{2}}\left|x_{i}-x_{j}\right|^{-1}\right. \\
& \left.-e \sum_{j<k \in \pi_{2}}\left|x_{j}-x_{k}\right|^{-1}-\sum_{i \in \pi_{1}} \delta_{i}(X)-e \sum_{j \in \pi_{2}} \delta_{j}(X)\right], \\
H^{(2)} & =\varepsilon \sum_{i=1}^{H}\left\{\left(p_{i}^{2}+m^{2}\right)^{1 / 2}-m\right\}-\kappa^{\prime} e[1+e M / L] \sum_{i=1}^{N} \delta_{i}(X) .
\end{aligned}
$$

In order that $(2.8)$ be an identity we require

$$
\left[2 e L M-e^{2} M(M-1)\right] \kappa^{\prime}=L(N-1) \kappa \text {. }
$$

The $N$ functions $\delta_{i}: \mathbb{R}^{3 N} \rightarrow \mathbb{R}$ are defined to be

$$
\delta_{i}(X)=\max \left\{\left|x_{j}-x_{i}\right|^{-1} \mid j \neq i\right\} .
$$

According to Corollary B.2 in the Appendix, $H^{(2)} \geqq-\varepsilon m N$ if the following condition among the parameters, which we shall assume, is satisfied.

$$
\varepsilon \geqq(\pi / 2) N^{1 / 3} \kappa^{\prime} e[1+e M / L] .
$$

Concerning the $h_{P}$ we note that they are all unitarily equivalent, so it suffices to study one of them. Call the first $L$ variables $Z=\left\{z_{1}, \ldots, z_{L}\right\}$ and the last $M$ variables $Y=\left\{y_{1}, \ldots, y_{M}\right\}$. Since there is no kinetic energy in $h_{P}$ associated with $Y$, the $y_{i}$ can be fixed. Furthermore, for $i=1, \ldots, L$

$$
\delta_{i}(X) \geqq \delta\left(z_{i} \mid Y\right) \equiv \max \left\{\left|z_{i}-y_{j}\right|^{-1} \mid 1 \leqq j \leqq M\right\}
$$

and, for $i=L+j$ with $1 \leqq j \leqq M, \delta_{i}(X) \geqq \delta_{j}(Y)$ (since $x_{L+j}=y_{j}$ by definition). Thus, if we define $h^{Y}$ on $q$ spin-state fermionic functions of $L$ variables by

$$
\begin{gathered}
h^{Y} \equiv(1-\varepsilon) \sum_{i=1}^{L}\left\{\left(p_{i}^{2}+m^{2}\right)^{1 / 2}-m\right\}-\kappa^{\prime} e \sum_{i=1}^{L} V^{Y}\left(z_{i}\right)+U^{Y} \\
U^{Y} \equiv \kappa^{\prime} e^{2}\left[\sum_{1 \leqq i<j \leqq M}\left|y_{i}-y_{j}\right|^{-1}+\sum_{j=1}^{M} \delta_{j}(Y)\right] \\
V^{Y}(z) \equiv \sum_{j=1}^{M}\left|z-y_{j}\right|^{-1}-\delta(z \mid Y)
\end{gathered}
$$

we have that for all $P$

$$
h_{P} \geqq \inf _{Y}\left\{\inf \operatorname{spec}\left(h^{Y}\right)\right\} .
$$

Lemma 1. If

$$
\frac{1}{2}>\varepsilon \geqq 1.7 q^{1 / 3}\left(\kappa^{\prime}\right)^{2 / 3} L^{1 / 3},
$$

then for all $Y$, and with $\kappa^{\prime \prime} \equiv(1-2 \varepsilon)^{-1} \kappa^{\prime}$,

$$
h^{Y} \geqq(1-2 \varepsilon) E_{\kappa^{\prime \prime}}^{C}(L)-\varepsilon m L .
$$


Proof. Let $B_{j}$ be the ball centered at $y_{j}$ of radius $r_{j}=\left[2 \delta_{j}(Y)\right]^{-1}$. These are pairwise disjoint. Let $\mu_{j}$ be the uniform normalized $\delta$-measure on $\partial B_{j}$, i.e. $\mu_{j}(x)=\left(4 \pi r_{j}^{2}\right)^{-1} \delta\left(\left|x-y_{j}\right|-r_{j}\right)$. Let $\mu=\sum_{j=1}^{M} \mu_{j}$. Let $\psi$ be any normalized fermionic $L$ particle function and $\varrho(x)$ its density [by (1.20) with $L$ in place of $N$ ].

Since $D(\varrho-e \mu, \varrho-e \mu) \geqq 0$, one easily derives [using $2 D\left(\mu_{j}, \mu_{j}\right)=r_{j}^{-1}, 2 D\left(\mu_{j}, \mu_{k}\right)$ $=\left|y_{j}-y_{k}\right|^{-1}$ and $\left(|x|^{-1} * \mu_{j}\right)(x)=\left|x-y_{j}\right|^{-1}$ for $\left.\left|x-y_{j}\right| \geqq r_{j}\right]$

$$
\kappa^{\prime} D(\varrho, \varrho) \geqq \kappa^{\prime} e \int \varrho(z) V^{Y}(z) d z-U^{Y} .
$$

Hence, $h^{Y}$ can be bounded by

$$
\begin{gathered}
\left(\psi, h^{Y} \psi\right) \geqq(1-2 \varepsilon) K^{Q}(\psi)-\kappa^{\prime} D(\varrho, \varrho)+\varepsilon K^{Q}(\psi), \\
K^{Q}(\psi) \equiv\left(\psi,\left[\sum_{i=1}^{L}\left(p_{i}^{2}+m^{2}\right)^{1 / 2}-m\right] \psi\right) .
\end{gathered}
$$

By Lemma B.3 (Appendix) with $g(x)=\xi^{3 / 4} \exp \left(-\pi \xi x^{2} / 2\right)$ and $(g,|p| g)=2 \xi^{1 / 2}$, we have

$$
\begin{gathered}
(1-2 \varepsilon)\left[K^{Q}(\psi)+2 \xi^{1 / 2} L\right]-\kappa^{\prime} D\left(\varrho * g^{2}, \varrho * g^{2}\right) \\
\geqq(1-2 \varepsilon) \mathscr{E}_{\kappa^{\prime \prime}}^{C}\left(\varrho * g^{2}\right) \geqq(1-2 \varepsilon) E_{\kappa^{\prime \prime}}^{C}(L) .
\end{gathered}
$$

The remainder terms which we have to bound below are

$$
R=-2(1-2 \varepsilon) \xi^{1 / 2} L+\varepsilon K^{Q}(\psi)-\frac{1}{2} \kappa^{\prime} \int \varrho(x) w(x-y) \varrho(y) d x d y
$$

with $w(x)=|x|^{-1}-\left(g^{2} *|x|^{-1} * g^{2}\right)(x)$. The integral in (2.25) can be bounded using Young's inequality by $\|w\|_{2}\|\varrho\|_{4 / 3}^{2}$. Clearly, $\|w\|_{2}=C \xi^{-1 / 4}$ with $C$ a constant; one easily finds $C^{2} \leqq 32 /\left(3 \pi^{1 / 2}\right)$. Optimizing the first and last terms in (2.25) with respect to $\xi$ (and replacing $1-2 \varepsilon$ by 1 ) we get

$$
R \geqq-(3 / 2) C^{2 / 3}\left(\kappa^{\prime}\right)^{2 / 3} L^{1 / 3}\|\varrho\|_{4 / 3}^{4 / 3}+\varepsilon K^{Q}(\psi) .
$$

But by (B.10), $K^{Q}(\psi) \geqq 1.6 q^{-1 / 3}\|\varrho\|_{4 / 3}^{4 / 3}-m L$ since $\left(p^{2}+m^{2}\right)^{1 / 2} \geqq|p|$. Thus, condition (2.19) implies $R \geqq-\varepsilon m L$.

Let us now put our results together to prove Theorem 1. There are five parameters $L, M$ (with $L+M=N), \varepsilon, e, \kappa^{\prime}$. These must satisfy (2.11), (2.13), (2.19). We set $e=L / M$ and determine $\kappa^{\prime}$ from (2.11), whence $\kappa^{\prime}<\kappa N / L$. Then we take

$$
\varepsilon=1.7 q^{1 / 3} \kappa^{2 / 3} N^{2 / 3} L^{-1 / 3}=1.7 q^{1 / 3} \tau^{2 / 3} N^{2 / 9} L^{-1 / 3}
$$

so that (2.19) is satisfied (as $N \rightarrow \infty, L / N \rightarrow 1$, so $\varepsilon<1 / 2$ ), The right side of (2.13) is less than $\pi \tau N^{2 / 3} / M$, so (2.13) will be satisfied [with (2.27)] if we choose

$$
M=1.9 q^{-1 / 3} \tau^{1 / 3} N^{7 / 9}
$$

Finally, $L=N-M=N\left(1-0\left(N^{-2 / 9}\right)\right)$ and $\varepsilon=O\left(N^{-1 / 9}\right)$.

Our lower bound $(2.8,2.20)$ is thus

$$
E_{\kappa}^{Q}(N) \geqq(N / L)(1-2 \varepsilon) E_{\kappa^{\prime \prime}}^{C}(L)-2 \varepsilon m N
$$


with $(1-2 \varepsilon) \kappa^{\prime \prime}=\kappa^{\prime}<\kappa N / L$. Since $N \rightarrow E^{C}(N)$ is concave and $E^{C}(0)=0$ (Sect. III), $(N / L) E^{C}(L) \geqq E^{C}(N)$, and thus

$$
E_{\kappa}^{Q}(N) \geqq N\left\{e^{c}\left(\tau^{\prime \prime}\right)-2 \varepsilon m\right\}
$$

with $\tau^{\prime \prime}=(1-2 \varepsilon)^{-1} \tau N / L=\tau+O\left(N^{-1 / 9}\right)$. This agrees with $N e^{c}(\tau)$ to $O\left(N^{8 / 9}\right)$ provided $\tau<\tau_{c}$.

\section{B.2. Lower Bound to the Quantum Energy (Bosons)}

The proof of Theorem 2 closely follows that of Theorem 1 just given, and only the differences will be mentioned below. Everything from (2.8) to (2.18) is the same. Condition (2.19) is not needed and the replacement for Lemma 1 is

Lemma 2. Let $\kappa^{\prime \prime}=(1-\varepsilon)^{-1} \kappa^{\prime}$. Acting on $L^{2}\left(\mathbb{R}^{3 N}\right)$ without statistics, $h^{Y}$ satisfies (for all $Y$ )

$$
h^{Y} \geqq(1-\varepsilon) E_{\kappa^{\prime \prime}}^{H}(L)
$$

The proof is the same as for Lemma 1 up to (2.22), but now we do not split $K^{Q}(\psi)$ into two pieces. We merely use Lemma B.5 which immediately yields (2.31).

Our only conditions are (2.11) and (2.13). As before, we set $e=L / M$ and determine $\kappa^{\prime}$ from (2.11), giving $\kappa^{\prime}<\kappa N / L$. To satisfy (2.13) we take

$$
\varepsilon=\pi N^{4 / 3} \kappa / M=\pi N^{1 / 3} \omega / M .
$$

(Recall $N \kappa=\omega$ for besons.) We take $M=N^{2 / 3}$ so that $\varepsilon=c N^{-1 / 3}$ and $L / N$ $=1-N^{-1 / 3}$. Then [again using $(N / L) E^{H}(L) \geqq E^{H}(N)$ ]

$$
E_{\kappa}^{Q}(N) \geqq N\left\{e^{H}\left(\omega^{\prime \prime}\right)-\varepsilon m\right\}
$$

with $\omega^{\prime \prime}=(1-\varepsilon)^{-1} \omega N / L$. This agrees with $N e^{H}(\omega)$ to $O\left(N^{2 / 3}\right)$ when $\omega<\omega_{c}$.

\section{Properties of Semiclassical Energies}

We begin with some a priori bounds on the components of the energy.

Lemma 3 (fermions). For any $\varrho \geqq 0$ with $\int \varrho=N \leqq N_{f}$ we have the following a priori estimates:

$$
\begin{gathered}
\int j(\varrho) d x+m N \leqq\left\{1-\left(N / N_{f}\right)^{2 / 3}\right\}^{-1}\left[\mathscr{E}^{C}(\varrho)+m N\right], \\
0 \leqq E^{C}(N)+m N \leqq C N\left[1-\left(N / N_{f}\right)^{2 / 3}\right]^{1 / 2},
\end{gathered}
$$

with $C=\frac{3}{2} m\left\{\int\left(\varrho_{F}\right)^{4 / 3} \int\left(\varrho_{F}\right)^{2 / 3}\right\}^{1 / 2}\left\|\varrho_{F}\right\|_{1}^{-1}$, where $\varrho_{F}$ is any minimizer for $F(\varrho)$ in (A.4). In particular, (3.2) implies that $E^{C}(N)$ is left continuous at $N_{f}$ and $E^{C}\left(N_{f}\right)=-m N_{f}$. When $N>N_{f}, E^{C}(N)=-\infty$.

Proof. Let $j_{0}(\varrho)=j(\varrho)+m \varrho$. By definition [and recalling $\gamma=\frac{3}{4}\left(6 \pi^{2} / g\right)^{1 / 3}$ ]

$$
m N+\mathscr{E}^{C}(\varrho)=\left(N / N_{f}\right)^{2 / 3}\left[\int j_{0}(\varrho)-\left(N_{f} / N\right)^{2 / 3} \kappa D(\varrho, \varrho)\right]+\left(1-\left(N / N_{f}\right)^{2 / 3}\right) \int j_{0}(\varrho) .
$$


Since $\int \varrho=N$ and $\kappa=\sigma_{f}^{-1} \gamma N_{f}^{-2 / 3}$ we have from the inequality $\int j_{0}(\varrho) \geqq \gamma \int \varrho^{4 / 3}$ that

$$
\begin{aligned}
m N+\mathscr{E}^{C}(\varrho) & \geqq\left(N / N_{f}\right)^{2 / 3} \gamma\left[\int \varrho^{4 / 3}-\sigma_{f}^{-1} N^{-2 / 3} D(\varrho, \varrho)\right]+\left(1-\left(N / N_{f}\right)^{2 / 3}\right) \int j_{0}(\varrho) \\
& \geqq\left(1-\left(N / N_{f}\right)^{2 / 3} \int j_{0}(\varrho) .\right.
\end{aligned}
$$

The last inequality follows from the definition of $\sigma_{f}$ in (A.5) and $\int \varrho=N$.

Let $\varrho_{\lambda}(x)=\lambda^{3} \varrho(\lambda x)$, where $\varrho \geqq 0$ is a fixed minimizer for $F(\varrho)$ in (A.4) with $\int \varrho=1$. By considering $\varrho_{\lambda}\left(N^{-1 / 3} x\right)$, we have from the variational principle that $m N+E^{C}(N) \leqq N \int j_{0}\left(\varrho_{\kappa}\right)-N^{5 / 3} \kappa D\left(\varrho_{\lambda}, \varrho_{\lambda}\right)$. Since $\left(p^{2}+m^{2}\right)^{1 / 2} \leqq|p|+m^{2} / 2|p|$, we find $j_{0}(\varrho) \leqq \gamma \varrho^{4 / 3}+\frac{9}{16} m^{2} \gamma^{-1} \varrho^{2 / 3}$. Using this,

$$
m N+E^{C}(N) \leqq N\left[\gamma \lambda \int \varrho^{4 / 3}+9 m^{2}(16 \gamma \lambda)^{-1} \int \varrho^{2 / 3}-\kappa N^{2 / 3} \lambda D(\varrho, \varrho)\right] .
$$

By definition, $D(\varrho, \varrho)=\sigma_{f} \int \varrho^{4 / 3}$ and $\kappa=\sigma_{f}^{-1} \gamma N_{f}^{-2 / 3}$, whence

$$
m N+E^{C}(N) \leqq N\left\{\left[1-\left(N / N_{f}\right)^{2 / 3}\right] \lambda \gamma \int \varrho^{4 / 3}+9 m^{2}(16 \gamma \lambda)^{-1} \int \varrho^{2 / 3}\right\} .
$$

Optimizing this with respect to $\lambda$ yields (3.2) and it also yields $E^{C}(N)=-\infty$ when $N>N_{f}$.

Lemma 4 (bosons). For any $\varrho \geqq 0$ and $\int \varrho=N \leqq N_{b}$, we have the following a priori estimates

$$
\begin{gathered}
\left(\varrho^{1 / 2},\left(p^{2}+m^{2}\right)^{1 / 2} \varrho^{1 / 2}\right) \leqq\left(1-N / N_{b}\right)^{-1}\left(\mathscr{E}^{H}(\varrho)+m N\right) \\
0 \leqq E^{H}(N)+m N \leqq C N\left[1-N / N_{b}\right]^{1 / 3},
\end{gathered}
$$

where $C=2 m\left(\varrho_{B}^{1 / 2},|p| \varrho_{B}^{1 / 2}\right)^{1 / 3}\left(\varrho_{B}^{1 / 2},|p|^{-1 / 2} \varrho_{B}^{1 / 2}\right)^{2 / 3}\left\|\varrho_{B}\right\|_{1}^{-1}$ and $\varrho_{B}^{1 / 2}$ is any minimizer for $B(\psi)$ in (A.6). Corollary A.2 implies that $C<\infty$. In particular, $E^{H}(N)$ is left continuous at $N_{b}$ and $E^{H}\left(N_{b}\right)=-m N_{b}$. When $N>N_{b}, E^{H}(N)=-\infty$.

Proof. Similar to Lemma 3, one uses $\left(p^{2}+m^{2}\right)^{1 / 2} \leqq|p|+m^{3 / 2}|p|^{-1 / 2}$.

In order to prove the concavity of $E^{C}(N)$ and $E^{H}(N)$, a technical lemma, whose proof is elementary, is needed.

Lemma 5. Suppose $f:[a, b] \rightarrow \mathbb{R}$ is a strictly decreasing concave function. Then for any constant $s>0$ the functions $g(t)=t f\left(s t^{2 / 3}\right)$ and $h(t)=t f(s t)$ are strictly concave on $\left[(a / s)^{3 / 2},(b / s)^{3 / 2}\right]$ and $[a / s, b / s]$ respectively.

Now we can begin the proofs of Theorems 3 and 4 .

Proof of Theorems $3(d)$ and $4(d) . e^{c}(\tau)$ in (1.13) is a strictly decreasing concave function because $e^{c}(\tau)=\inf \{$ non-constant linear functions of $\tau$ \}. The strict concavity of $E^{C}(N)$ and $E^{H}(N)$ follows from the scaling relations $(1.14,1.15)$ and Lemma 5. The continuity of $E^{C}(N)$ and $E^{H}(N)$ at $N_{f}$ and $N_{b}$ is in Lemmas 3 and 4 .

Proof of Theorems 3(a), (b), (c), (e). The existence of a minimizer was first proved by Auchmuty and Beals [1]. The fact that a minimizing $\varrho$ is necessarily symmetric decreasing follows from the strong rearrangement inequality in [13] (see Appendix A.1).

Part (b) is a standard result in the calculus of variations. 
To prove (c) we use the a-priori estimate (3.2) which reads (under the assumption of a minimizing $\varrho$ when $\left.N=N_{f}\right)-m N_{f}=E^{C}\left(N_{f}\right)=\mathscr{E}^{C}(\varrho)$. Since $\left(p^{2}+m^{2}\right)^{1 / 2}-m>|p|-m$, we have generally (with $\int \varrho=N$ )

$$
j(\varrho)>\gamma \varrho^{4 / 3}-m N .
$$

Thus, in our case, $-m N_{f}>A(\varrho)-m N_{f}$ with $A(\varrho)=\gamma \int \varrho^{4 / 3}-\kappa D(\varrho, \varrho)$. But when $N=N_{f}=\tau_{c}^{3 / 2} \kappa^{-3 / 2}, A(\varrho) \geqq 0$ [see (1.9) and (A.5)]. This is a contradiction.

As for (e), the concavity of $E^{C}(N)$ implies the existence of left-hand and righthand derivations for all $N$, and they are equal a.e. Part (e) follows by considering $t \varrho_{N}$ as a variational function, differentiating $\mathscr{E}^{C}\left(t \varrho_{N}\right)$ at $t=1+$ and $1-$, and using the Euler-Lagrange equation (1.16).

Proof of Theorem 4(a), (b), (c), (e). The proof is essentially the same as that for Theorem 3 except for the existence part (a). Here the proof is virtually the same as that for Choquard's equation in [13]. Note that Lemma 4 places an a-priori bound on the kinetic energy which permits us to use a weak compactness argument. The key fact is in (A.2) [and also (A.1)] which permits us to restrict a minimizing sequence to symmetric decreasing functions. Note that the weak compactness argument leads to the existence of a function $\bar{\varrho}_{N}$ satisfying $\int \bar{\varrho}_{N} \leqq N$ and $\mathscr{E}^{H}\left(\bar{\varrho}_{N}\right)$ $\leqq E^{H}(N)$. Since $E^{H}(N)$ is strictly monotone decreasing in $N$, we must have equality in both cases.

To prove Theorems 3(f) and 4(f), Lemmas 6 and 7 (which are intrinsically different in the two cases) are needed.

Lemma 6 (fermions). Let $\varrho$ and $\mu>0$ satisfy (1.16) with $\int \varrho=N$. We do not assume $\varrho$ is a minimizer for $N$, but we do assume $\varrho$ is radial, i.e. $\varrho(x)=\varrho(r), r=|x|$. Then

$$
\begin{gathered}
\mathscr{E}^{C}(\varrho)=\int\left\{4 j(\varrho)-3 \varrho j^{\prime}(\varrho)\right\} d x, \\
K(\varrho) \equiv \int j(\varrho) d x=\frac{5}{2} \mathscr{E}^{C}(\varrho)+\frac{3}{2} \mu N .
\end{gathered}
$$

Proof. Multiply (1.17) by $r^{3} \varrho(r)$ and integrate. Then $-4 \pi \int_{0}^{\infty} r^{3} j^{\prime \prime}(\varrho) \varrho \varrho d r$ $=4 \pi \kappa \int_{0}^{\infty} M(r) \varrho(r) r d r$. But the second integrals is $\kappa D(\varrho, \varrho)$ (by Newton's theorem). The first integrals is $-4 \pi \int r^{3} \frac{d}{d r}\left(\varrho j^{\prime}(\varrho)-j(\varrho)\right) d r$. After integrating by parts [and using $\left.\left(\varrho j^{\prime}-j\right)(0)=0\right]$ it becomes $3 \int\left\{\varrho j^{\prime}(\varrho)-j(\varrho)\right\} d x$. This proves (3.6) since $\mathscr{E}^{C}(\varrho)$ $=K(\varrho)-\kappa D(\varrho, \varrho)$. To prove (3.7), multiply (1.16) by $\varrho(x)$ and integrate. Then $\int \varrho j^{\prime}(\varrho) d x=2 \kappa D(\varrho, \varrho)-\mu N$. Combining this with (3.6) yields (3.7).

Remark. (3.6) is a virial theorem. It can also be proved for minimizing $\varrho$ 's by replacing $\varrho(x)$ by $\varrho_{\lambda}(x)=\lambda^{3} \varrho(\lambda x)$ and differentiating $\mathscr{E}^{C}\left(\varrho_{\lambda}\right)$ with respect to $\lambda$ at $\lambda=1$.

Lemma 7 (bosons). Suppose $\varrho(x) \geqq 0, \varrho^{1 / 2} \in L^{2}\left(\mathbb{R}^{3}\right),\left(\varrho^{1 / 2},|p| \varrho^{1 / 2}\right)<\infty$ and $\varrho$ satisfies (1.19) for some $v$ (in the sense of distributions). Let $\int \varrho=N$. Then

$$
K(\varrho) \equiv\left(\varrho^{1 / 2},\left\{\left(p^{2}+m^{2}\right)^{1 / 2}-m\right\} \varrho^{1 / 2}\right)=2 \mathscr{E}^{H}(\varrho)+v N .
$$


Proof. Since $\left(\varrho^{1 / 2},|p| \varrho^{1 / 2}\right)<\infty$ and $\varrho^{1 / 2} \in L^{2}$, we have $\int \varrho\left(|x|^{-1} * \varrho\right)<$ (const) $\|\varrho\|_{6 / 5}^{2}$ $<\infty$ by Young's and Sobolev's inequalities. Multiply (1.19) by $\varrho^{1 / 2}$ and integrate. This yields (3.8). Note that although (1.19) holds only in $\mathscr{D}^{\prime}$, this integration is justified since all the terms are separately finite.

Proof of Theorems $3(f), 4(f)$. We prove the boson case using Lemma 7 . The fermion proof is the same using Lemma 6. Assume, on the contrary, that there is a sequence $N \rightarrow N_{b}$ with minimizers $\varrho_{N}$ satisfying (1.19) with $v_{N}$. Suppose that $v_{N} \rightarrow \infty$. Then since $\mathscr{E}^{H}\left(\varrho_{N}\right)=\mathrm{E}^{H}(\mathrm{~N}) \rightarrow \mathrm{E}^{H}\left(\mathrm{~N}_{b}\right)=-\mathrm{mN}_{b}$ [Theorem $4(\mathrm{~d})$ ], we see from Lemma 7 that $K\left(\varrho_{N}\right)$ is bounded. Recall in the proof of Theorem 4(a) that the proof of the existence of a minimizer for any $N$ needed a bound on $K(\varrho)$ for a minimizing sequence. Formerly we used Lemma 4 to achieve this when $N<N_{b}$. But now, by our assumption on $v_{N}$, we also have uniform boundedness of $K\left(\varrho_{N}\right)$. By the proof of Theorem 4(a) we have a function $\bar{\varrho}$ (weak limit of $\varrho_{N}$ ) with $\int \bar{\varrho} \leqq N_{b}$ and $\mathscr{E}^{H}(\bar{\varrho})=$ $-m N_{b}$. As in Theorem 4(a), this implies that $\varrho$ is a minimizer for $N=N_{b}$ and this contradicts Theorem 4(c).

\section{Properties of the Semiclassical Density (Fermions)}

Our main goal here is to prove the uniqueness of the minimizer of the semiclassical functional for each $N<N_{f}$. This will enable us to prove Theorem 5 . The main facts about the density $\varrho_{N}$ are summarized in Fig. 2, which will be explained later. We shall explore all radial [i.e. $\varrho(x)=\varrho(|x|)$ ] solutions to $(1.16)$; this class includes all minimizers by Theorem 3 . Henceforth $\varrho$ will be assumed to be radial without further mention. We shall also suppress irrelevant constants [by replacing $\varrho(x)$ by $a \varrho(b x)$ ] so that (1.16) becomes

$$
j^{\prime}(\varrho(x))=\left[\left(|x|^{-1} * \varrho\right)(x)-\mu\right]_{+}
$$

with $j^{\prime}(t)=\left(t^{2 / 3}+1\right)^{1 / 2}-1$. The side condition is $\int \varrho=N<N_{f}=\left(3 / 4 \sigma_{f}\right)^{3 / 2}$ in these units [see (A.5) and Lemma 3]. We also have defined

$$
M(r)=4 \pi \int_{0}^{r} \varrho(s) s^{2} d s
$$

so that

$$
V_{\varrho} \equiv|x|^{-1} * \varrho=r^{-1} M(r)+4 \pi \int_{r}^{\infty} t \varrho(t) d t
$$

Equation (4.1) implies

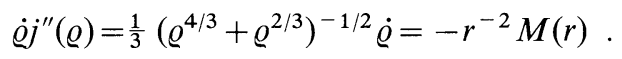

\section{A. Regularity Properties}

Since $V_{\varrho}(r) \approx N / r$ as $r \rightarrow \infty$ we see, first of all, that $\mu>0$, for otherwise for large $r, \varrho \approx(N / r)^{3 / 2} \notin L^{1}$. By Newton's theorem, (4.1) implies that $\varrho$ has compact support in a ball of radius

$$
R=N / \mu
$$


and, since $V_{\varrho}(r)$ is continuous in $r$ and $j^{\prime}(\varrho)$ is continuous in $\varrho$ with $j^{\prime}(0)=0$, we have that $\varrho(r) \rightarrow 0$ as $r \rightarrow R$. Since $d V_{\varrho} / d r<0$, in the domain $B_{R}=\{r \mid r<R\}$ we can write (4.1) as

$$
j^{\prime}(\varrho(x))=V_{\varrho}(x)-\mu, \quad \text { in } \quad B_{R}
$$

with $\varrho(R)=0$. We also see [since $j^{\prime}(\varrho)$ is monotone] that $\varrho$ is necessarily monotone decreasing. Since $V_{\varrho}(r)<N / r$ we can easily iterate (4.6), starting with $j^{\prime}(\varrho)<N / r-\mu$, to conclude that $\varrho$ is $C^{\infty}$ in $B_{R}$. (Here we have to use the fact that $\left(j^{\prime}\right)^{-1}(t)$ $=\left(t^{2}+2 t\right)^{3 / 2}$ is $C^{\infty}$ for $\left.t>0\right)$.

By applying $\Delta$ to (4.6) we get

$$
-\Delta \Theta=4 \pi\left(j^{\prime}\right)^{-1}(\Theta)=4 \pi\left[\Theta^{2}+2 \Theta\right]^{3 / 2}
$$

with $\Theta=V_{\varrho}-\mu \geqq 0$. Equation (4.7) holds on $B_{R}$ with the boundary condition $\Theta(R)=0$. Notice that in this version of the problem $N$ is not mentioned. By [22, Theorem 5.8.6] we can go one step further and assert that $\Theta$ (and hence $\varrho$ ) is real analytic in $B_{R}$.

Since $\varrho$ is radial, (4.7) is an ODE. We are looking for a strong solution to (4.7), with $\Theta(0)=\beta<\infty$ and $\dot{\Theta}(0)=0$. Starting with these initial conditions (with $\beta$ arbitrary) we can easily (using Picard's method for example) prove that (4.7) has a unique positive solution for each $\beta$ up to some $R=R(\beta)$ at which $\Theta(R)$ vanishes (which conceivably could be $R=\infty$ ).

It is clear that every strong solution to (4.7) with the property that $R(\beta)<\infty$ gives a solution to (4.6). Conversely, a solution to (4.6) gives a solution to (4.7) with $\beta=j^{\prime}(\varrho(0))$. However, it is conceivable that for some $\beta>0$, the solution to (4.7) has $R(\beta)=\infty$. Later on, by an indirect argument, we will show that this does not happen, but it seems worthwhile to give a direct proof now.

Lemma 8. Let $\Theta$ be the radial solution to (4.7) obtained by integrating outwards from $r=0$ with the initial conditions $\Theta(0)=\beta$ and $\dot{\Theta}(0)=0$. Then there is some $0<R(\beta)<\infty$ such that $\Theta(R(\beta))=0$. For $r<R(\beta), \dot{\Theta}(r)<0$.

Proof. Integrating (4.7) twice we get, for $r \leqq R(\beta)$,

$$
\Theta(r)=\beta-4 \pi \int_{0}^{r}\left(t^{-1}-r^{-1}\right) t^{2} \varrho(t) d t
$$

with $\varrho=\left(\Theta^{2}+2 \Theta\right)^{3 / 2}$. From (4.8) it is clear that $\dot{\Theta}(r)<0$. Suppose $R(\beta)=\infty$. We first claim $g(t) \equiv t \varrho(t) \in L_{r / 2}^{1}([0, \infty), d r)$. This follows from $\int_{0}^{r}\left(t^{-1}-r^{-1}\right) t g(t) d t$ $\geqq \int_{0}^{r / 2}\left(t^{-1}-r^{-1}\right) \operatorname{tg}(t) d t \geqq \frac{1}{2} \int_{0}^{r / 2} g(t) d t ;$ thus, if $g(t) \notin L^{1}$ we would have from (4.8) that $\Theta(r)<0$ for large $r$. Next, by Proposition 9 below, $r^{-1} \int_{0}^{r} \operatorname{tg}(t) \rightarrow 0$ as $r \rightarrow \infty$. Then, from (4.8), as $r \rightarrow \infty, \Theta(r) \rightarrow \beta-C$ with $C=4 \pi \int_{0}^{\infty} g(t) d t$. Since $\Theta(r) \geqq 0$, $\beta \geqq C$. Hence $\Theta(r) \geqq 4 \pi r^{-1} \int_{0}^{r} \operatorname{tg}(t) d t \geqq C^{\prime} / r$ for large $r$. But, since $\varrho \geqq(2 \Theta)^{3 / 2}$, we have that $\varrho>C^{\prime \prime} r^{-3 / 2}$ for large $r$ and hence $\int_{0}^{\infty} g(t) d t=\infty$, which is a
contradiction. $\square$ 
Proposition 9. Suppose $f:[0, \infty) \rightarrow \mathbb{R}^{+}$and $\int_{0}^{\infty} f(t) d t=C<\infty$. Then $r^{-1} \int_{0}^{r} t f(t) d t \rightarrow 0$
as $r \rightarrow \infty$. as $r \rightarrow \infty$

Proof. Let $I(r)=\int_{0}^{r} t f(t) d t$. Choose $1>\varepsilon>0$ and write $I(r)=\int_{0}^{r \varepsilon} t f(t) d t+\int_{r \varepsilon}^{r} t f(t) d t$. The first integral is bounded by $r \varepsilon \int_{0}^{r \varepsilon} f(t) d t \leqq r \varepsilon C$. The second is bounded by $r K_{\varepsilon}(r)$ with $K_{\varepsilon}(r)=\int_{r \varepsilon}^{r} f(t) d t$. Since $f \in L^{1}, \quad K_{\varepsilon} \rightarrow 0$ as $r \rightarrow \infty$. Thus $\lim \sup r^{-1} I(r)$ $\leqq \varepsilon C+\lim K_{\varepsilon}(r)=\varepsilon C$. This holds for all $\varepsilon$, and thus proves the Proposition.

Let us pause to summarize the situation. For each choice of the central density $\varrho(0)$, which we henceforth call $\alpha\left(\alpha=\left(\beta^{2}+2 \beta\right)^{3 / 2}\right)$ there is (by Lemma 8$)$ a unique radial solution that satisfies (4.1) for some unique $\mu=\mu(\alpha)>0$. This $\varrho$ is real analytic up to $R=N(\alpha) / \mu(\alpha)$, where $N(\alpha)=\int \varrho$. The qualitative nature of this $\varrho$ is shown in Fig. 2.

Suppose that $N(\alpha)$ were a strictly monotone increasing function of $\alpha$. Then, since a minimizing $\varrho$ satisfies (4.1) and is radial we would conclude: (i) All radial solutions of (4.1) are minimizers; (ii) for each $N$ the minimizing $\varrho$ is unique. But we do not yet know that $N(\alpha)$ is strictly monotone increasing, and that is the problem we now address. Up to this point the arguments were fairly standard (with the possible exception of Lemma 8) and that is why we were brief.

\section{B. Uniqueness and Comparison Properties of Minimizers}

Our strategy will be to first focus on solutions to (4.1) which are minimizers for $\mathscr{E}^{C}$. Then we will show that all solutions to (4.1) are minimizers.

Lemma 10. Suppose $\varrho_{1}$ and $\varrho_{2}$ are minimizers for $\mathscr{E}^{C}$ with $\int \varrho_{1}=N_{1}, \int \varrho_{2}=N_{2}$ respectively. Let $R_{1}$ and $R_{2}$ be the radii of their supports and let $R=\max \left(R_{1}, R_{2}\right)$. Suppose that $\varrho_{1}(0)>\varrho_{2}(0)$. [If $\varrho_{1}(0)=\varrho_{2}(0)$ then $\varrho_{1} \equiv \varrho_{2}$ and this is uninteresting.] Then for all $0<r<R$ we have $M_{1}(r)>M_{2}(r)$ [see (4.2)].

Proof. Suppose on the contrary, there is an $r_{0}$ such that $M_{1}\left(r_{0}\right) \leqq M_{2}\left(r_{0}\right)$. Since $M_{1}(r)>M_{2}(r)$ for $r$ sufficiently small, there exists (by continuity) an $\tilde{r}, 0<\tilde{r} \leqq r_{0}$, such that $M_{1}(\tilde{r})=M_{2}(\tilde{r}) \equiv Q$ (say). We first note that $\varrho_{1}(\tilde{r}) \neq \varrho_{2}(\tilde{r})$. Otherwise from (4.4) we would have $\dot{\varrho}_{1}(\tilde{r})=\dot{\varrho}_{2}(\tilde{r})$ which together with $\varrho_{1}(\tilde{r})=\varrho_{2}(\tilde{r})$ would imply $\Theta_{1}(\tilde{r})=\Theta_{2}(\tilde{r})$ and $\dot{\Theta}_{1}(\tilde{r})=\dot{\Theta}_{2}(\tilde{r})$. By the uniqueness theorem of ODE, $\Theta_{1} \equiv \Theta_{2}$ which

is a contradiction.
Now define the set (for $\left.N_{j} \geqq Q \geqq 0, j=1,2\right) \mathscr{A}_{Q}\left(N_{j}\right)=\left\{\varrho \geqq 0 \mid \int_{|x| \leqq \tilde{r}} \varrho=Q\right.$ and $\left.\int_{|x| \geq \tilde{r}} \varrho=N_{j}-Q\right\}$ which is subset of $\mathscr{A}\left(N_{j}\right)=\left\{\varrho \geqq 0 \mid \int \varrho=N_{j}\right\}$. From the variational principle, $E^{C}\left(N_{1}\right) \leqq \inf _{\varrho \in \mathscr{A}_{Q}\left(N_{1}\right)} \mathscr{E}^{C}(\varrho) \equiv E_{Q}\left(N_{1}\right)$. But since $\varrho_{1} \in \mathscr{A}_{Q}\left(\mathrm{~N}_{1}\right)$ by assumption, we have in fact $E^{C}\left(N_{1}\right)=E_{Q}\left(N_{1}\right)$. Similarly $E^{C}\left(N_{2}\right)=E_{Q}\left(N_{2}\right)$. Now define the following sets (with $i, o$ denoting inside and outside)

$$
\begin{gathered}
\mathscr{A}^{i}=\left\{\varrho^{i} \geqq 0 \mid \varrho^{i}(x)=0 \text { if }|x|>\tilde{r} \text { and } \int \varrho^{i}=Q\right\} \\
\mathscr{A}_{j}^{o}=\left\{\varrho^{o} \geqq 0 \mid \varrho^{o}(x)=0 \text { if }|x| \leqq \tilde{r} \text { and } \int \varrho^{o}=N_{j}-Q\right\} .
\end{gathered}
$$


It is easy to check that $S: \mathscr{A}^{i} \times \mathscr{A}_{j}^{o} \rightarrow \mathscr{A}_{Q}\left(N_{j}\right)$ defined by $S\left(\varrho^{i}, \varrho^{o}\right)=\varrho^{i}+\varrho^{o}$ is a bijection. Define a new functional on $\mathscr{A}_{j}^{o}$

$$
\mathscr{E}_{Q}^{o}\left(\varrho^{o}\right)=\int j\left(\varrho^{o}\right)-D\left(\varrho^{o}, \varrho^{o}\right)-Q \int|x|^{-1} \varrho^{o}(x) d x .
$$

Let $\mathscr{E}_{Q}^{i}\left(\varrho^{i}\right) \equiv \mathscr{E}^{C}\left(\varrho^{i}\right)$. Then, for any $\varrho \in \mathscr{A}_{Q}\left(N_{j}\right)$ it is easy to check that [with $\left.S^{-1}(\varrho)=\varrho^{i}, \varrho^{o}\right] \mathscr{E}^{C}(\varrho)=\mathscr{E}_{Q}^{i}\left(\varrho^{i}\right)+\mathscr{E}_{Q}^{o}\left(\varrho^{o}\right)$, where we have used Newton's theorem and the definition of $Q=\int_{|x| \leqq \tilde{r}} \varrho$.

Let $E_{Q}^{i}=\inf \left\{\mathscr{E}_{Q}^{i}\left(\varrho^{i}\right) \mid \varrho^{i} \in \mathscr{A}^{i}\right\}$ and $E_{Q, j}^{o}=\inf \left\{\mathscr{E}_{Q}^{o}\left(\varrho^{o}\right) \mid \varrho^{o} \in \mathscr{A}_{j}^{o}\right\}$. Then by the variational principle, we have $E_{Q}\left(N_{j}\right)=E_{Q}^{i}+E_{Q, j}^{o}$. Then, since $\varrho_{j}$ is a minimizer for $E^{C}\left(N_{j}\right), \mathscr{E}_{Q}^{i}\left(\varrho_{j}^{i}\right)+\mathscr{E}_{Q}^{o}\left(\varrho_{j}^{o}\right)=\mathscr{E}^{C}\left(\varrho_{j}\right)=E_{Q}^{i}+E_{Q, j}^{o}$. Since $E_{Q}^{i} \leqq \mathscr{E}_{Q}^{i}\left(\varrho_{j}^{i}\right)$ and $E_{Q}^{o}\left(\varrho_{j}^{o}\right) \leqq \mathscr{E}_{Q}^{o}\left(\varrho_{j}^{o}\right)$ by the variational principle, we obtain $E_{Q}^{i}=\mathscr{E}_{Q}^{i}\left(\varrho_{j}^{i}\right)$ and $E_{Q, j}^{o}=\mathscr{E}_{Q}^{o}\left(\varrho_{j}^{o}\right)$. Now let $\tilde{\varrho} \equiv \varrho_{1}^{i}$ $+\varrho_{2}^{o}$. It is easy to check that $\tilde{\varrho}$ is also a minimizer for $N_{2}$ by using $\mathscr{E}_{Q}^{i}\left(\varrho_{2}^{i}\right)=\mathscr{E}_{Q}^{i}\left(\varrho_{1}^{i}\right)$. But $\tilde{\varrho}$ is not continuous at $\tilde{r}$, which violates the regularity of the minimizer proved above. Another way to reach a contradiction is to note that $\varrho \equiv \varrho_{2}^{i}+\varrho_{1}^{o}$ is a minimizer for $N_{1}$. One of the two functions, $\varrho$ and $\varrho$ must be increasing at $\tilde{r}$, and this contradicts the symmetric decreasing property of minimizers.

Remarks. (a) The same method and conclusion apply to minimizers for some other functionals $\mathscr{E}(\varrho)$ which can be written as $\mathscr{E}(\varrho)=\int j(\varrho(x)) d x-D(\varrho, \varrho)$.

(b) Lemma 10 does not say $M_{1}(R)>M_{2}(R)$. In fact, we shall later see that this is true, but we do not yet know it. If we knew in advance that $M_{1}(R)>M_{2}(R)$ the proof of the following Lemma 11 would be trivial.

Lemma 11. There exist at most one minimizing $\varrho$ for $E^{C}(N)$ when $N<N_{f}$.

Proof. Suppose, on the contrary, that we have two minimizers $\varrho_{1}$ and $\varrho_{2}$ with $\varrho_{1}(0)>\varrho_{2}(0)$. Let $R_{1}$ and $R_{2}$ be the radii of their supports. By Lemma 10 , $M_{1}(r)>M_{2}(r)$ for all $0<r<\max \left(R_{1}, R_{2}\right)$. Then $R_{1} \leqq R_{2}$, for otherwise $N=M_{2}\left(R_{2}\right)$ $<M_{1}\left(R_{2}\right)<N$. Let $J(z)=4 j(z)-3 j^{\prime}(z) z$, whence $J(z)$ is concave since $J^{\prime}(z)=-1$ $+\left(z^{2 / 3}+1\right)^{-1 / 2}$ is decreasing. From (3.6), $0=\mathscr{E}^{C}\left(\varrho_{1}\right)-\mathscr{E}^{C}\left(\varrho_{2}\right)=\int\left[J\left(\varrho_{1}\right)-J\left(\varrho_{2}\right)\right] d x$ $=4 \pi \int_{0}^{R_{2}}\left[J\left(\varrho_{1}(r)\right)-J\left(\varrho_{2}(r)\right)\right] r^{2} d r \leqq 4 \pi \int_{0}^{R_{2}} r^{2}\left(\varrho_{1}(r)-\varrho_{2}(r)\right) J^{\prime}\left(\varrho_{2}(r)\right) d r . \quad$ The last inequality is a consequence of the concavity of $J$. Integrating the last integral by parts and using the definition of $M(r)$, we have $0 \leqq-\int_{0}^{R_{2}}\left(M_{1}(r)\right.$ $\left.-M_{2}(r)\right) J^{\prime \prime}\left(\varrho_{2}(r)\right) \varrho_{2}(r) d r$. Since $J^{\prime \prime}(z)<0, \dot{\varrho}_{2}(r) \leqq 0$ and $M_{1}(r)>M_{2}(r)$, this last integral is negative, which is a contradiction.

Remark. Note that the only property of $j(z)$ used in the above proof is the concavity of $J(z)$. Since the concavity of $J(z)$ is equivalent to the convexity of $z \rightarrow j^{\prime}\left(z^{3}\right)$, Lemma 11 holds for all functionals with $j^{\prime}\left(z^{3}\right)$ convex.

Lemma 10 says that if $\varrho_{1}(0)>\varrho_{2}(0)$ then $N_{1} \geqq N_{2}$. But Lemma 11 say that $N_{1}=N_{2}$ is impossible if $\varrho_{1}$ is not identical to $\varrho_{2}$. Therefore we have

Corollary 2. If $\varrho_{1}$ and $\varrho_{2}$ are minimizers for $N_{1}$ and $N_{2}$ respectively and if $\varrho_{1}(0)>\varrho_{2}(0)$ then $N_{1}>N_{2}$. 
Lemma 12. Let $\varrho^{\alpha}$ be the unique bounded nonnegative solution of (4.1) with central density $\alpha=\varrho(0)$. Then $\varrho$ is the unique minimizer for $E^{C}(N)$ with $N=\int \varrho^{\alpha}$. In other words, all the solutions of (4.1) parametrized by their central density are in fact minima of $E^{C}(N)$ for some $N$.

Proof. Let $G=\left(0, N_{f}\right)$ and let $D=\left\{\alpha \mid \varrho^{\alpha}\right.$ is a minimizer for some $\left.N \in G\right\}$. For each $N \in G$ there is a unique minimizing $\varrho_{N}$, and hence a unique central density $\alpha_{N}$. We let $\Gamma: G \rightarrow D$ denote this map from $N \in G$ to $\alpha_{N}$.

(i) $\Gamma$ is $1: 1$ by the aforementioned uniqueness of the ODE (4.7) with given initial condition $\alpha$.

(ii) $\Gamma^{-1}$ is continuous on $D$ and $D$ is closed in $\mathbb{R}^{+}$. To prove this we suppose $\alpha_{j} \rightarrow \alpha \in \mathbb{R}^{+}$monotonically. By Corollary $2, N_{j}=\Gamma^{-1}\left(\alpha_{j}\right)$ is monotone and bounded, so $N_{j}$ has a limit $N$. It is clear that $N<N_{f}$ for otherwise $\mu_{j} \rightarrow \infty$ [Theorem 3(f)] but $V_{j}(r)$ is uniformly bounded [since $\varrho_{j}(r)<\alpha_{j}$ and $\int \varrho_{j}<\mathrm{N}_{f}$ ]; these facts would contradict (4.1) when $r$ is small. By continuity of the solution of the ODE with respect to $\alpha$ (this follows e.g. from the contraction mapping principle) $\varrho_{j}(r) \rightarrow \varrho(r)$ pointwise and $\varrho(r)$ satisfies (4.7) with $\varrho(0)=\alpha$. We have to prove that $\varrho$ is a minimizer for $N$, which will imply $\alpha \in D$ (whence $D$ is closed) and $\Gamma^{-1}$ is continuous. By (3.1) $\varrho_{j}$ is uniformly bounded in $L^{4 / 3}$ so (by passing to a subsequence) $\varrho_{j} \rightarrow \varrho$ weakly in $L^{4 / 3}$. Then $\mathscr{E}^{C}(\varrho) \leqq \lim \mathscr{E}^{C}\left(\varrho_{j}\right)=\lim E^{C}\left(N_{j}\right)=E^{C}(N)$. [The lower semicontinuity, namely $\mathscr{E}^{C}(\varrho) \leqq \lim \mathscr{E}^{C}\left(\varrho_{j}\right)$, follows as in [13], for example. Clearly $\int j(\varrho)$ $\leqq \lim \int j\left(\varrho_{j}\right)$ since $\varrho_{j} \rightarrow \varrho$. On the other hand $D\left(\varrho_{j}, \varrho_{j}\right) \rightarrow D(\varrho, \varrho)$ since $\varrho$ is symmetric decreasing and $\varrho_{j} \rightarrow \varrho$ pointwise.] Thus, $\varrho$ is a minimizer for $N$ because $\int \varrho \leqq N$ while $E^{C}(N)$ is strictly decreasing; therefore $\int \varrho=N$.

(iii) $\Gamma^{-1}$ is $1: 1$ and continuous from $D$ onto $G$ and also $D$ is closed in $\mathbb{R}^{+}$. Hence $\Gamma^{-1}$ is a homeomorphism from $D$ to $G$. Since $G$ is connected, so is $D$, and therefore $D$ is a closed interval in $\mathbb{R}^{+}$. Since the only closed interval in $\mathbb{R}^{+}$homeomorphic to $\left(0, N_{f}\right)$ is $\mathbb{R}^{+}$itself, we conclude that $D=\mathbb{R}^{+}$.

Finally we want to make a qualitative comparison of solutions with different $N$. First a technical lemma is needed.

Lemma 13. Suppose $\Theta_{1}$ and $\Theta_{2}$ are two nonnegative solutions of (4.7) with radii $R_{1}$ and $R_{2}$ with $R_{1}<R_{2}$. Then there exists an $\tilde{r}<R_{1}$ such that $\Theta_{1}(\tilde{r}) \geqq \Theta_{2}(\tilde{r})$.

Proof. This is a standard Sturm comparison argument. Assuming, on the contrary, that $\Theta_{2}(r)>\Theta_{1}(r)$ for all $r<R_{1}$, let $u(r)=\Theta_{1}(r) / \Theta_{2}(r)$. Then, from (4.7), $u$ satisfies $\Theta_{2} \Delta u+2 \nabla u \cdot \nabla \Theta_{2}+\left(F\left(\Theta_{1}\right)-F\left(\Theta_{2}\right)\right) \Theta_{2} u=0$ with $F(t)=4 \pi\left(t^{2}+2 t\right)^{1 / 2}(t+2)$. Now $u\left(R_{1}\right)=0$ and $0 \leqq u(r)<1$ for $r<R_{1}$. Hence $g \equiv F\left(\Theta_{1}\right)-F\left(\Theta_{2}\right)<0$. At a maximum, $\Delta u \leqq 0, \nabla u=0$, but this contradicts $g<0$.

Lemma 14. Let $\varrho_{1}$ and $\varrho_{2}$ be two solutions to (4.1) with central densities $\alpha_{1}>\alpha_{2}$. Then $R_{1}<R_{2}$ and there is precisely one point $r$ in $\left(0, R_{1}\right)$ at which $\varrho_{1}(r)=\varrho_{2}(r)$ (as in Fig. 2$)$.

Proof. We know $M_{1}(r)>M_{2}(r)$ when $0<r \leqq R \equiv \max \left(R_{1}, R_{2}\right)$. Suppose $R_{1} \geqq R_{2}$. Then integrate (4.4) from $r<R_{2}$ to $R_{2}$ for both $\varrho_{1}$ and $\varrho_{2}$. One has $j^{\prime}\left(\varrho_{i}(r)\right)$ $-j^{\prime}\left(\varrho_{i}\left(R_{2}\right)\right)=-\int_{r}^{R_{2}} s^{-2} M_{i}(s) d s$ for $i=1,2$. Since $j^{\prime}\left(\varrho_{1}\left(R_{2}\right)\right) \geqq 0=j^{\prime}\left(\varrho_{2}\left(R_{2}\right)\right)$ and $M_{1}(s)>M_{2}(s)$ for $0<s<R_{2}$, we easily conclude $j^{\prime}\left(\varrho_{1}(r)\right)>j^{\prime}\left(\varrho_{2}(r)\right)$ and hence 
$\varrho_{1}(r)>\varrho_{2}(r)$ for $0 \leqq r<R_{2}$. If $R_{1}>R_{2}$, then this contradicts Lemma 13, so suppose $R_{1}=R_{2}=R$. Then, similar to Lemma 13 , define $u=\Theta_{2} / \Theta_{1}$. By Gauss's theorem $\dot{\Theta}_{j}(R)=N_{j} R^{2}$, since $\int \varrho_{j}=N_{j}$. Then $u(R)=N_{2} / N_{1} \equiv \delta<1$. As in Lemma 13 (using $\varrho_{1} \geqq \varrho_{2}$ ), $u$ can have no maximum for $r<R$. Thus $u \leqq \delta$. However, $N_{2}$ $=\int\left(\Theta_{2}^{2}+2 \Theta_{2}\right)^{3 / 2} \leqq \int\left(\delta^{2} \Theta_{1}^{2}+2 \delta \Theta_{1}\right)^{3 / 2} \leqq \delta^{3 / 2} \int\left(\Theta_{1}^{2}+2 \Theta_{1}\right)^{3 / 2}=\delta^{3 / 2} N_{1}$, so $\delta \geqq 1$, which is a contradiction. Thus, $R_{1}<R_{2}$.

Since $\alpha_{1}>\alpha_{2}$ the function $f \equiv \varrho_{1}-\varrho_{2}$ which is $C^{\infty}$ for $t<R_{1}$ must have at least one zero in $\left(0, R_{1}\right)$. Suppose there is more than one zero. At each zero we have, by (4.4) that $d f / d r<0$ (since $M_{1}>M_{2}$ ). But it is easy to see that a $C^{1}$ function cannot have a negative derivative at all its zeros.

\section{Summary}

Let us summarize the results of A. and B., for this is Theorem 5 .

(i) All radial solutions of the Euler-Lagrange equation (4.1) are in $L^{1}$ and are minimizers.

(ii) They are real analytic up to the cut off radius $R$. For $r \approx R, \Theta(r) \approx R-r$ so $\varrho(r) \approx(R-r)^{3 / 2}$ as in Fig. 2.

(iii) They are parametrized by the central density $\alpha$ which goes from 0 to $\infty . \mu(\alpha)$ and $N(\alpha)$ are strictly monotone increasing while $R(\alpha)$ is strictly monotone decreasing. As $\alpha \rightarrow \infty, \mu(\alpha) \rightarrow \infty, N(\alpha) \rightarrow N_{f}, R(\alpha) \rightarrow 0$. As $\alpha \rightarrow 0, N(\alpha) \rightarrow 0, R(a) \rightarrow \infty$ [to be proved in (vii) below], and hence $\mu(\alpha)=N(\alpha) / R(\alpha) \rightarrow 0 . N, \mu, R$ are continuous in $\alpha$.

(iv) Any two solutions always have exactly one intersection as in Fig. 2.

(v) For each $r$, the mass $M(r)$ is an increasing function of $\alpha$.

(vi) Proof of Theorem 5(b). In the notation of Theorem 3(e), suppose that $\left(d E^{C} / d N\right)_{+} \neq\left(d E^{C} / d N\right)_{-}$for some $N<N_{f}$. In this case it is easy to see that $\mu_{N}$ must be discontinuous at $N$. However, by (4.5) $\mu_{N}$ is continuous since $N \rightarrow \alpha_{N}$ is continuous (by Lemma 12) and $\alpha \rightarrow R(\alpha)$ is continuous [by continuity of the solution of (4.7) with respect to the "initial data" at $r=0]$.

(vii) Proof of Theorem 5(c). The only fact yet to be proved is that $R_{N} \rightarrow \infty$ as $N \rightarrow 0$ [or equivalently, $R(\alpha) \rightarrow \infty$ as $\alpha \rightarrow 0$ ]. Suppose on the contrary, $R(\alpha)<R_{0}$ for all $\alpha$. Choose a solution $\Theta_{1}$ of the Lane-Emden equation

$$
\Delta \Theta+4 \pi(2 \Theta)^{3 / 2}=0
$$

with $\Theta_{1}\left(R_{1}\right)=0$ for some $R_{1}>2 R_{0}$ and $\Theta_{1}(0)<1$. Such a $\Theta_{1}$ always exists since there is a scaling $\Theta(x) \rightarrow \lambda^{4} \Theta(\lambda x)$. Let $\beta \equiv \frac{1}{8} \Theta_{1}\left(R_{0}\right)$ and let $\Theta_{\beta}$ be the solution of (4.7) with $\Theta_{\beta}(0)=\beta$. Then $\Theta_{\beta}(r)<\frac{1}{8} \Theta_{1}(r)$ and $\left(\Theta_{\beta}^{2}+2 \Theta_{\beta}\right)^{1 / 2}\left(\Theta_{\beta}+2\right)<\left(2 \Theta_{1}\right)^{1 / 2}$ for $0 \leqq r \leqq R_{0}$ [since $\Theta_{1}$ and $\Theta_{\beta}$ are monotone decreasing and $\Theta_{1}\left(R_{0}\right)=8 \Theta_{\beta}(0)$ ]. But this is impossible as can be seen by the argument given in Lemma 13.

\section{Convergence of the Quantum Density to the Semiclassical Density (Fermions)}

Here we prove Theorem 6 for fermions. As explained in Sect. I, we first add a fixed single-particle potential $\lambda \chi_{\tau}\left(N^{-1 / 3} x\right)$ to $H_{\kappa N}$. (Recall that $\chi_{\tau}$ is the characteristic function of the support of $\varrho_{\tau}$.) Following the method in [18] we next add an 
additional one parameter single-particle potential $\delta W\left(N^{-1 / 3} x\right)$ and differentiate with respect to $\delta$ at $\delta=0$. Two facts have to be established: (i) an extension of Theorem 1 to include the potential $\lambda \chi+\delta W$; (ii) the differentiability of the corresponding semiclassical energy with respect to $\delta$. We shall call (i) Theorem $1^{\prime}$ and (ii) Lemma 15.

For any $W \in C_{0}^{\infty}\left(\mathbb{R}^{3}\right)$ and $\delta \in \mathbb{R}$, define [with $H_{\kappa N \lambda}$ defined in (1.22)]

$$
\begin{gathered}
H_{\kappa N \lambda \delta} \equiv H_{\kappa N \lambda}+\delta \sum_{i=1}^{N} W\left(N^{-1 / 3} x_{i}\right), \\
E_{\lambda \delta}^{Q}(N)=\inf \operatorname{spec} H_{\kappa N \lambda \delta}, \\
\varepsilon_{\tau \lambda \delta}^{c}(\varrho)=\varepsilon_{\tau}^{c}(\varrho)-\lambda \int \chi_{\tau} \varrho+\delta \int W \varrho, \\
e_{\lambda \delta}^{c}(r)=\inf \left\{\varepsilon_{\tau \lambda \delta}^{c}(\varrho) \mid \varrho \geqq 0, \int \varrho=1\right\} .
\end{gathered}
$$

Theorem 1' (fermions). Fix $q, \lambda$ and $\tau=\kappa N^{2 / 3}$ with $\tau<\tau_{c}$. Then $\lim N^{-1} E_{\lambda \dot{\lambda}}^{Q}(N)$ $=e_{\lambda \delta}^{c}(\tau)$.

Proof. (a) Upper Bound. Let $\bar{\varrho}=\varrho * g^{2}$ with $g(x)=\xi^{3 / 4} \exp \left(-\pi \xi x^{2} / 2\right)$. Then we have generally $G \equiv\left|\int(\varrho(x)-\bar{\varrho}(x)) W\left(N^{-1 / 3} x\right) d x\right| \leqq \int \varrho\left\|W * \bar{g}^{2}-W\right\|_{\infty}$, where $\bar{g}(x)$ $=\bar{\xi}^{3 / 4} \exp \left(-\pi \bar{\xi} x^{2} / 2\right)$ with $\bar{\xi}=N^{2 / 3} \xi$. By the same method as in the proof of Theorem 1 , we only have to show that $N^{-1}\left[1.68 \kappa \int \varrho^{4 / 3}+2 N \xi^{1 / 2}+\xi^{-1} \kappa \int \varrho^{2}+G\right] \rightarrow 0$ as $N \rightarrow \infty$. Note that the minimizing $\xi$ for (2.2) is $\xi=\kappa^{2 / 3}$, whence $\bar{\xi}=N^{2 / 9} \tau^{2 / 3} \rightarrow \infty$. This implies $N^{-1} G=\left\|W * \bar{g}^{2}-W\right\|_{\infty} \rightarrow 0$ as $N \rightarrow \infty$.

(b) Lower Bound. We only have to show that [cf. (2.25)] $N^{-1} R^{\prime} \equiv N^{-1}[R+G]$ $\geqq N^{-1}\left[-2 \xi^{1 / 2} L+\varepsilon K^{Q}(\psi)-C \kappa^{\prime} \xi^{-1 / 4}\|\varrho\|_{4 / 3}^{2}-G\right]_{-} \rightarrow 0$ as $N \rightarrow \infty$ (with $|f|_{-}$ $=\min (0, f))$. Let $\xi=\max \left\{L^{-4 / 3}\|\varrho\|_{4 / 3}^{8 / 3}\left(\kappa^{\prime}\right)^{4 / 3}, N^{-1 / 3}\right\}$. Then $N^{2 / 3} \xi \equiv \bar{\xi} \geqq N^{1 / 3}$ whence, as in the proof of part (a), $G / N \rightarrow 0$ as $N \rightarrow \infty$. We take $\varepsilon$ and $\kappa^{\prime}$ exactly the same as in the proof of Theorem 1. For the first three terms, we can use the same estimate as in Lemma 1 if $\xi=L^{-4 / 3}\|\varrho\|_{4 / 3}^{8 / 3}\left(\kappa^{\prime}\right)^{4 / 3}$. If $\xi=N^{-1 / 3}$ we have that $\|\varrho\|_{4 / 3}$ $\leqq($ const $) N^{17 / 24}$, and then the result is immediate.

Lemma 15. Fix $\lambda$ and $\tau<\tau_{c}$. Then $e_{\lambda \delta}^{c}(\tau)$ is differentiable at $\delta=0$ and $d e_{\lambda \delta}^{c}(\tau) /\left.d \delta\right|_{\delta=0}$ $=\int \varrho_{\tau} W$.

Proof. For each $\delta>0$ choose a function $\varrho_{\delta}$ satisfying $\delta^{-1}\left|\varepsilon_{\tau \lambda \delta}^{c}\left(\varrho_{\delta}\right)-e_{\lambda \delta}^{c}(\tau)\right| \rightarrow 0$ as $\delta \rightarrow 0$. By the variational principle, $\delta \int \varrho_{\tau} W \geqq e_{\lambda \delta}^{c}(\tau)-e_{\lambda}^{c}(\tau) \geqq \delta \int \varrho_{\delta} W+e_{\lambda \delta}^{c}(\tau)$ $-\varepsilon_{\tau \lambda \delta}^{c}\left(\varrho_{\delta}\right)$. To prove the lemma we only have to show that $\int \varrho_{\delta} W \rightarrow \int \varrho_{\tau} W$ as $\delta \rightarrow 0$. Clearly, $e_{\lambda \delta}^{c}(\tau)$ is continuous in $\delta$ and therefore, by the assumption about $\varrho_{\delta}$, we see that $\varrho_{\delta}$ is a minimizing sequence, as $\delta \rightarrow 0$, for $e_{\lambda}^{c}(\tau)=e^{c}(\tau)-\lambda$. Since $\varepsilon_{\tau}^{c}\left(\varrho_{\delta}\right) \geqq \varepsilon_{\tau}^{c}\left(\varrho_{\delta}\right)$ $-\lambda$, we have that $\varrho_{\delta}$ is also a minimizing sequence for $e^{c}(\tau)$ and $\int \varrho_{\delta} \chi_{\tau} \rightarrow 1$. We also know that the minimizer, $\varrho_{\tau}$, for $e^{c}(\tau)$ is unique if we center it at the origin (Theorem 5). By Lion's result [20] there exists a sequence $y_{\delta} \in \mathbb{R}^{3}$ such that $\varrho_{\delta}\left(x+y_{\delta}\right) \rightarrow \varrho_{\tau}(x)$ strongly in $L^{4 / 3} \cap L^{1}$. But since $\int \chi_{\tau} \varrho_{\delta} \rightarrow 1$ we must have $y_{\delta} \rightarrow 0$.

Proof of Theorem 6. For any approximate ground state $\psi_{N \lambda}$ we have, from the variational principle, that

$$
\delta \int \varrho_{N \lambda}^{Q}\left(N^{1 / 3} x\right) W(x) d x \geqq N^{-1}\left\{E_{\lambda \delta}^{Q}(N)-E_{\lambda}^{Q}(N)+E_{\lambda}^{Q}(N)-\left(\psi_{N \lambda}, H_{N \lambda} \psi_{N \lambda}\right)\right\} .
$$


By taking the limit $N \rightarrow \infty$ and then $\delta \rightarrow 0$ (using Theorem $1^{\prime}$ and Lemma 15), $\lim _{N \rightarrow \infty} \int \varrho_{N \lambda}^{Q}\left(N^{1 / 3} x\right) W(x) d x=\int \varrho_{\tau}(x) W(x)$ for any $W \in C_{0}^{\infty}\left(\mathbb{R}^{3}\right)$. Hence $\varrho_{N \lambda}^{Q}\left(N^{1 / 3} x\right)$ $\rightarrow \varrho_{\tau}(x)$ in weak $L^{4 / 3}\left(\mathbb{R}^{3}\right)$. The fact that $\varrho_{N \lambda}^{Q}\left(N^{1 / 3} x\right) \rightarrow \varrho_{\tau}(x)$ also in weak $L^{1}\left(\mathbb{R}^{3}\right)$ follows form Lemma III.4 of [18].

\section{Appendix A: Some Definitions and Basic Facts}

\section{A.1. Symmetric Decreasing Rearrangements}

Given a function $\psi: \mathbb{R}^{3} \rightarrow \mathbb{C}$, the symmetric decreasing rearrangement $\psi^{*}$ of $\psi$ satisfies $\psi^{*}: \mathbb{R}^{3} \rightarrow \mathbb{R}^{+}, \psi^{*}(x)$ depends only on $|x|$ and Lebesgue meas $\left\{x \mid \psi^{*}(x)>a\right\}$ $=$ Lebesgue meas $\{x|| \psi(x) \mid>a\}$ for all $a>0$. $\psi$ is symmetric decreasing if $\psi=\psi^{*}$. It follows that if $j: \mathbb{R}^{+} \rightarrow \mathbb{R}^{+}$and $\varrho(x) \geqq 0$ then $\int j\left(\varrho^{*}\right) d x=\int j(\varrho) d x$. Also $\left(\psi^{*}\right)^{2}=\left(\psi^{2}\right)^{*}$. A particular case of the Riesz inequality states that

$$
\iint \varrho(x) \varrho(y)|x-y|^{-1} d x d y \leqq \iint \varrho^{*}(x) \varrho^{*}(y)|x-y|^{-1} d x d y,
$$

and the strict rearrangement inequality in [13] states that (A.1) is a strict inequality unless $\varrho(x)=\varrho^{*}(x-y)$ for some $y \in \mathbb{R}^{3}$.

We also have that

$$
\begin{aligned}
(\psi,|p| \psi) & \geqq\left(\psi^{*},|p| \psi^{*}\right), \\
(\psi, T \psi) & \geqq\left(\psi^{*}, T \psi^{*}\right),
\end{aligned}
$$

with $T=\left(p^{2}+m^{2}\right)^{1 / 2}-m=\left(-\Delta+m^{2}\right)^{1 / 2}-m$. This follows from the proof in [13] and the fact that the kernels $e^{-t|p|}(x, y)$ and $e^{-t T}(x, y)$ are symmetric decreasing functions of $x-y$, as shown in [4].

\section{A.2. Minimizers for the Gravitational Energy}

In the fermion case we are concerned with the ratio (for $\varrho \in L^{4 / 3} \cap L^{1}$ )

$$
F(\varrho)=D(\varrho, \varrho)\|\varrho\|_{4 / 3}^{-4 / 3}\|\varrho\|_{1}^{-2 / 3}
$$

which is homogeneous in $\varrho$ and dilation invariant. We set

$$
\sigma_{f} \equiv \sup F(\varrho)=1.092 \text {. }
$$

It is a known fact (proved in [17]) that there is a minimizing $\varrho \equiv \varrho_{F}$ for $F(\varrho)$. It is unique up to scaling, dilation and translation: $\varrho_{F}(x) \rightarrow a \varrho_{F}(b x+y)$. It satisfies Emden's equation. The value 1.092 in (A.5) is numerical.

For bosons we consider the ratio

$$
\begin{aligned}
B(\psi) & =D\left(|\psi|^{2},|\psi|^{2}\right)(\psi,|p| \psi)^{-1}\|\psi\|_{2}^{-2}, \\
\sigma_{b} & \equiv \sup \left\{B(\psi) \mid \psi \in L^{2},(\psi,|p| \psi)<\infty\right\} .
\end{aligned}
$$

By (B.10) with $N=1,(\psi,|p| \psi) \geqq C\|\psi\|_{8 / 3}^{2}$ [actually, there is a Sobolev ineqality $\left.(\psi,|p| \psi) \geqq C\|\psi\|_{3}^{2}\right]$. This, together with the Hardy-Littlewood-Sobolev inequality, $D(\varrho, \varrho) \leqq C\|\varrho\|_{6 / 5}^{2}$, shows that $B(\varrho)$ is bounded. In fact, the rearrangement inequalities (A.1), (A.2) permit us to imitate the proof in [17] and show that there 
is a minimizing $\psi$ for $B(\psi)$ with $\psi \in L^{2}$ and $(\psi,|p| \psi)<\infty$. See also the proof of Theorem 4(a). We have no precise numerical value for $\sigma_{b}$. However, Theorem 2 states that $\omega_{c}=1 / \sigma_{b}$ and, using the results in $[19, \mathrm{pp} .503,504]$ we have that $\pi / 4>\sigma_{b}$ $>1 / 2.7$.

Another fact we shall need is

Lemma A.1. Let $\psi$ be any minimizer for $B(\psi)$. Then $\psi \in L^{3 / 2+\varepsilon}$, all $\varepsilon>0$.

Proof. Note that $\psi=\psi^{*}$ and satisfies

$$
|p| \psi=\{W(x)-v\} \psi
$$

with $W=$ (const) $|x|^{-1} *|\psi|^{2}$ and $v>0$. Equation (A.8) is just the Euler-Lagrange equation. Since $|\psi|^{2}=\left|\psi^{2}\right|^{*}$ and $|\psi|^{2} \in L^{1}, W(x) \rightarrow 0$ as $|x| \rightarrow \infty$. Thus, writing $f \equiv\{W(x)-v\} \psi$ and using $\psi(x) \geqq 0$, we have that $f_{+}$has compact support in a ball of some radius $R$. Since

$$
\psi=|p|^{-1}\{|p| \psi\}=\left(2 \pi^{2}\right)^{-1}|x|^{-2} * f \leqq\left(2 \pi^{2}\right)^{-1}|x|^{-2} * f_{+}
$$

we see that $\psi(x)<c|x|^{-2}$ for $|x|>R$.

Corollary A.2. If $\psi$ is a minimizer for $B(\psi)$ then $\left(\psi,|p|^{-1 / 2} \psi\right)<\infty$.

Proof. $|p|^{-1 / 2}$ is convolution with $|x|^{-5 / 2}$. By the Hardy-Littlewood-Sobolev inequality $\psi \in L^{12 / 7} \Rightarrow\left(\psi,|p|^{-1 / 2} \psi\right)<\infty$.

\section{Appendix B : Some Inequalities}

\section{B.1. Domination of the Nearest Neighbor Coulomb Potential by $|p|$}

Let $Y=\left\{y_{1}, \ldots, y_{N}\right\}$ be $N$ arbitrary, but fixed points in $\mathbb{R}^{3}$ and let $Z=\left\{z_{1}, \ldots, z_{N}\right\}$ with $z_{j} \in \mathbb{R}, z_{j} \geqq 0$ be given. Define

$$
\bar{z}=\|z\|_{3}=\left\{\sum z_{j}^{3}\right\}^{1 / 3} .
$$

For $x \in \mathbb{R}^{3}$, the function

$$
\omega_{Y Z}(x) \equiv \max _{j}\left|x-y_{j}\right|^{-1} z_{j}
$$

can be called the nearest neighbor Coulomb potential. Let

$$
Q=\left\{\left.f\left|f \in L^{2}\left(\mathbb{R}^{3}\right), \int\right| \hat{f}(p)\right|^{2}|p| d p<\infty\right\},
$$

where $\hat{f}(p)=\int f(x) \exp ($ ip $\cdot x) d x$ denotes Fourier transform.

Lemma B.1. For all $Y$ and $Z$ and $f \in Q$

$$
(2 \pi)^{-3} \int|\widehat{f}(p)|^{2}|p| d p \equiv(f,|p| f) \geqq \frac{2}{\pi}(\bar{z})^{-1}\left(f, \omega_{Y Z} f\right)
$$

Proof. If $N=1$ the lemma is known [8-10,26] and we shall reduce (B.4) to that case. Let $f^{*}$ denote the symmetric decreasing rearrangement of $f$. By (A.2) $(f,|p| f) \geqq\left(f^{*},|p| f^{*}\right)$. On the other hand $\left(f, \omega_{Y Z} f\right) \leqq\left(f^{*}, \omega_{Y Z}^{*} f^{*}\right)$. We claim that 
$\omega_{Y Z}^{*}(x) \leqq \bar{z}|x|^{-1}$, from which (B.4) follows by applying the $N=1$ lemma to $f^{*}$. To prove this it is only necessary to note that for all $b>0$ (with $\mu=$ Lebesgue measure)

$$
\begin{aligned}
\mu\left\{x \mid \omega_{Y Z}(x)>b\right\} & \leqq \sum_{j=1}^{N} \mu\left\{x\left|z_{j}\right| x-\left.y_{j}\right|^{-1}>b\right\} \\
& =(4 \pi / 3) b^{-3} \sum_{j=1}^{N} z_{j}^{3}=\mu\left\{\left.x|\bar{z}| x\right|^{-1}>b\right\} .
\end{aligned}
$$

Corollary B.2. Let $\psi: \mathbb{R}^{3 N} \rightarrow \mathbb{C}$ be an $N$ particle function (without any particular statistics) in $Q^{N}$. Let $\delta_{i}: \mathbb{R}^{3 N} \rightarrow \mathbb{R}$ denote the $N$ functions defined in (2.12), i.e. $\delta_{i}(X)=\max \left\{\left|x_{i}-x_{j}\right|^{-1} \mid j \neq i\right\}$. Then for each $i$

$$
\left(\psi,\left|p_{i}\right| \psi\right) \geqq(2 / \pi)(N-1)^{-1 / 3}\left(\psi, \delta_{i} \psi\right)
$$

Inequality (B.6) holds without regard to statistics. If, on the other hand, $\psi$ is restricted to be a $q$-state fermionic function, it is possible to prove that

$$
\sum_{i=1}^{N}\left(\psi,\left|p_{i}\right| \psi\right) \geqq(\text { const }) q^{-1 / 3} \sum_{i=1}^{N}\left(\psi, \delta_{i} \psi\right) .
$$

Since (B.7) is not needed here we defer its proof to a forthcoming paper of ours. Fefferman and de la Llave [6] have proved (B.7) for $q=1$ but their method does not appear to be easily generalizable to $q>1$.

\section{B.2. Semiclassical Lower Bounds to the Kinetic Energy}

The single particle kinetic energy operator is $T=\left(p^{2}+m^{2}\right)^{1 / 2}-m$ with $p^{2}=-\Delta$. Let $\psi$ be a normalized wave function for $N$ fermions with $q$ spin states each and let

$$
K^{Q}(\psi)=\left(\psi, \sum_{i=1}^{N} T_{i} \psi\right)
$$

be the total kinetic energy. The semiclassical approximation to $K^{Q}(\psi)$ is

$$
K^{Q}(\psi) \approx K\left(\varrho_{\psi}\right) \equiv \int j\left(\varrho_{\psi}(x)\right) d x
$$

with $\varrho_{\psi}$ being the single particle density defined by $(1.20)$ and with $j(t)$ given by (1.3).

It is conjectured that $K\left(\varrho_{\psi}\right)$ is, in fact, a lower bound to $K^{Q}(\psi)$ but no one has proved this. Daubechies [4], using the method in [12], has found two lower bounds of the right form for fermions, the first of which we use here.

$$
\begin{gathered}
\left(\psi,\left\{\sum_{i=1}^{N}\left|p_{i}\right|\right\} \psi\right) \geqq 1.6 q^{-1 / 3} \int \varrho_{\psi}(x)^{4 / 3} d x, \\
K^{Q}(\psi) \geqq C \int j\left(C^{-1} \varrho_{\psi}(x)\right) d x
\end{gathered}
$$

with $C=9.6$.

Although (B.10) is important for us, we also need a bound similar to (B.9). This is provided by 
Lemma B.3 (fermions). Let $g \in Q$ [cf. (B.3)] with $\|g\|_{2}=1$. Then, for all normalized $\psi$,

$$
K^{Q}(\psi) \geqq \int j\left(\left(\varrho_{\psi} *|g|^{2}\right)\right) d x-N(g,|p| g) .
$$

Proof. This is the same as that given in [15, Eqs. (5.14)-(5.22)]. Introduce the coherent states $g_{p q} \in L^{2}\left(\mathbb{R}^{3}\right)$ by $g_{p q}(x)=g(x-q) \exp (i p \cdot x)$. Here $p, q \in \mathbb{R}^{3}$. Let $\pi_{p q}$ be the projection onto $g_{p q}$. Then for $f \in L^{2}\left(\mathbb{R}^{3}\right)$

$$
\begin{gathered}
(f, f)=(2 \pi)^{-3} \iint\left(f, \pi_{p q} f\right) d p d q \\
\left(f,\left(V *|g|^{2}\right) f\right)=(2 \pi)^{-3} \iint V(q)\left(f, \pi_{p q} f\right) d p d q \\
(f, T f) \geqq(2 \pi)^{-3} \iint \tilde{T}(p)\left(f, \pi_{p q} f\right) d p d q-\|f\|_{2}^{2}(g,|p| g) .
\end{gathered}
$$

Here, $\widetilde{T}(p)=\left(p^{2}+m^{2}\right)^{1 / 2}-m$ is a function, not an operator. Equations (B.13) and (B.14) are obvious. Inequality (B.15) is easily proved by writing $f$ as a Fourier integral and then using (2.1) with $k \rightarrow p, p \rightarrow r-p$. Thus, if $h$ is the operator $T-\left(V *|g|^{2}\right)(x)$ and $\gamma$ is a positive semidefinite operator with $\operatorname{Tr} \gamma=\lambda$ we have (by making an eigenvector expansion of $\gamma$ ) that

$$
\operatorname{Tr} \gamma h \geqq(2 \pi)^{-3} \iint d p d q[\tilde{T}(p)-V(q)] M(p, q)-\lambda(g,|p| g)
$$

with $M(p, q)=\operatorname{Tr} \gamma \pi_{p q} \geqq 0$. If, in addition, $0 \leqq \gamma \leqq q I$ then $0 \leqq M(p, q) \leqq q$ and hence

$$
\begin{aligned}
& \operatorname{Tr} \gamma h \geqq-(2 \pi)^{-3} q \iint d p d q[V(q)-\tilde{T}(p)]_{+}-\lambda(g,|p| g) \\
& =-\left(q / 6 \pi^{2}\right) \int\left(V^{2}+2 m V\right)^{3 / 2} V+\int j\left(\left(q / 6 \pi^{2}\right)\left(V^{2}+2 m V\right)^{3 / 2}\right)-\lambda(g,|p| g) .
\end{aligned}
$$

Recall that $j(t)$ depends on $q$.

Let $\varrho(x) \equiv \gamma(x, x)$ and take $\left(V^{2}+2 m V\right)^{3 / 2}=\left(6 \pi^{2} / q\right) \varrho *|g|^{2}$. Noting that $\operatorname{Tr} \gamma\left(V *|g|^{2}\right)=\int V\left(\varrho *|g|^{2}\right)$, (B.17) becomes

$$
\operatorname{Tr} \gamma T \geqq \int j\left(\varrho *|g|^{2}\right)-\lambda(g,|p| g) .
$$

To apply this to our case, let $\gamma$ be given by the kernel

$$
\begin{aligned}
\gamma(x, y)= & N \sum_{\sigma} \int \psi\left(x, x_{2}, \ldots, x_{N} ; \sigma_{1}, \ldots, \sigma_{N}\right) \\
& \cdot \bar{\psi}\left(y, x_{2}, \ldots, x_{N}, \sigma_{1}, \ldots, \sigma_{N}\right) d x_{2} \ldots d x_{N} .
\end{aligned}
$$

Then $\gamma \geqq 0, \lambda=\operatorname{Tr} \gamma=N$. The fact that $\gamma \leqq q I$ is standard [16]. Inequality (B.18) becomes (B.12).

Lemma B. 3 can trivially be generalized to operators other than $T$ and to $n \neq 3$ in the following way.

Lemma B.4. Let $\tilde{T}: \mathbb{R}^{n} \rightarrow \mathbb{R}^{+}$satisfy $\tilde{T}(p) \geqq 0$ and, for all $p, q \in \mathbb{R}^{n}, \tilde{T}(p) \leqq \widetilde{T}(p-q)$ $+\widetilde{S}(q)$ for a suitable nonnegative function $S: \mathbb{R}^{n} \rightarrow \mathbb{R}^{+}$. Let $T$ and $S$ be the corresponding multiplication operators in momentum space. Let $g: \mathbb{R}^{n} \rightarrow \mathbb{C}$ with $\|g\|_{2}=1$. Then

$$
\left(\psi, \sum T_{i} \psi\right) \geqq \int J\left(\left(\varrho_{\psi} *|g|^{2}\right)(x)\right) d x-N(g, S g)
$$


Here, $J: \mathbb{R}^{+} \rightarrow \mathbb{R}^{+}$is defined as follows. Let

Then

$$
\begin{aligned}
& \mu(t)=q(2 \pi)^{-n} \text { meas }\{p \mid \tilde{T}(p) \leqq t\} \\
& \beta(t)=q(2 \pi)^{-n} \int_{\tilde{T}(p) \leqq t} \tilde{T}(p) d p .
\end{aligned}
$$

$$
J(t)=\beta\left(\mu^{-1}(t)\right)
$$

where $\mu^{-1}$ is the inverse function. (It is not always uniquely defined everywhere, but $\beta \circ \mu^{-1}$ is.)

On the other hand, for bosons (or, more generally, particles without any statistics) (B.9) is not a good approximation to $K^{Q}(\psi)$. The following is a good approximation and also a bound.

Lemma B.5 (bosons).

$$
K^{Q}(\psi) \geqq\left(\varrho_{\psi}^{1 / 2}, T \varrho_{\psi}^{1 / 2}\right)
$$

The proof of this is that given by Conlon for Lemma 4.2 in [3]. One only has to verify that $e^{-t T}$ has a positive kernel, but this is proved in [4, Remark 1].

Acknowledgements. The authors are grateful to Christopher King and Michael Loss for helpful discussions about this work.

\section{References}

1. Auchmuty, J., Beals, R.: Variational solutions of some nonlinear free boundary problems. Arch. Ration. Mech. Anal. 43, 255-271 (1971). See also Models of rotating stars. Astrophys. J. 165, L79-L82 (1971)

2. Chandrasekhar, S. : Phil. Mag. 11, 592 (1931); Astrophys. J. 74, 81 (1931); Monthly Notices Roy. Astron. Soc. 91, 456 (1931); Rev. Mod. Phys. 56, 137 (1984)

3. Conlon, J.: The ground state energy of a classical gas. Commun. Math. Phys. 94, 439-458 (1984)

4. Daubechies, I.: An uncertainty principle for fermions with generalized kinetic energy. Commun. Math. Phys. 90, 511-520 (1983)

5. Daubechies, I., Lieb, E.H.: One-electron relativistic molecules with Coulomb interaction. Commun. Math. Phys. 90, 497-510 (1983)

6. Fefferman, C., de la Llave, R. : Relativistic stability of matter. I. Rev. Math. Iberoamericana 2, 119-215 (1986)

7. Fowler, R.H.: Monthly Notices Roy. Astron. Soc. 87, 114 (1926)

8. Herbst, I. : Spectral theory of the operator $\left(p^{2}+m^{2}\right)^{1 / 2}-z e^{2} / r$. Commun. Math. Phys. 53, 285-294 (1977); Errata ibid. 55, 316 (1977)

9. Kato, T.: Perturbation theory for linear operators. Berlin, Heidelberg, New York: Springer 1966. See Remark 5.12, p. 307

10. Kovalenko, V., Perelmuter, M., Semenov, Ya.: Schrödinger operators with $L_{w}^{1 / 2}\left(\mathbb{R}^{l}\right)$ potentials. J. Math. Phys. 22, 1033-1044 (1981)

11. Landau, L.: Phys. Z. Sowjetunion 1, 285 (1932)

12. Lieb, E.: The number of bound states of one-body Schrödinger operators and the Weyl problem. Proc. Am. Math. Soc. Symp. Pure Math. 36, 241-252 (1980). See also: Bounds on the eigenvalues of the Laplace and Schrödinger operators. Bull. Am. Math. Soc. 82, $751-753(1976)$ 
13. Lieb, E.: Existence and uniqueness of the minimizing solution of Choquard's nonlinear equation. Stud. Appl. Math. 57, 93-105 (1977)

14. Lieb, E.: Variational principle for many-fermions systems. Phys. Rev. Lett. 46, 457-459 (1981); Errata ibid. 47, 69 (1981)

15. Lieb, E.: Thomas-Fermi and related theories of atoms and molecules. Rev. Mod. Phys. 53, 603-641 (1981); Errata ibid. 54, 311 (1982)

16. Lieb, E.: Density functionals for Coulomb systems. Int. J. Quant. Chem. 24, 243-277 (1983)

17. Lieb, E., Oxford, S.: An improved lower bound on the indirect Coulomb energy. Int. J. Quant. Chem. 19, 427-439 (1981)

18. Lieb, E., Simon, B.: Thomas Fermi theory of atoms, molecules and solids. Adv. Math. 23, 22 $116(1977)$

19. Lieb, E., Thirring, W.: Gravitational collapse in quantum mechanics with relativistic kinetic energy. Ann. Phys. (NY) 155, 494-512 (1984)

20. Lions, P.-L.: The concentration compactness principle in the calculus of variations; the locally compact case I. Ann. Inst. H. Poincaré Anal. non lineaire 1, 109-145 (1984)

21. Messer, J. : Lecture Notes in Physics, Vol. 147. Berlin, Heidelberg, New York: Springer 1981

22. Morrey, C.B., Jr.: Multiple integrals in the calculus of variations, Theorem 5.8.6. Berlin, Heidelbereg, New York: Springer 1966

23. Ni, W.M.: Uniqueness of solutions of nonlinear Dirichlet problems. J. Differ. Equations 5, 289-304 (1983)

24. Straumann, S.: General relativity and relativistic astrophysics. Berlin, Heidelberg, New York: Springer 1984

25. Thirring, W.: Bosonic black holes. Phys. Lett. B127, 27 (1983)

26. Weder, R. : Spectral analysis of pseudodifferential operators. J. Funct. Anal. 20, 319-337 (1975)

27. Weinberg, S.: Gravitation and cosmology. New York: Wiley 1972

28. Hamada, T., Salpeter, E.: Models for zero temperature stars. Astrophys. J. 134, 683 (1961)

29. Shapiro, S., Teukolsky, S.: Black holes, white dwarfs and neutron stars. New York: Wiley 1983

30. Ruffini, R., Bonazzola, S.: Systems of self-gravitating particles in general relativity and the concept of an equation of state. Phys. Rev. 187, 1767-1783 (1969)

Communicated by E.H. Lieb

Received March 2, 1987, in revised form March 27, 1987 